ARTICLE

httpst//doi.org/10.1038/s414.67-020-17277-3

\title{
"Gene accordions" cause genotypic and phenotypic heterogeneity in clonal populations of Staphylococcus aureus
}

Darya Belikova', Angelika Jochim', Jeffrey Power ${ }^{1}$, Matthew T. G. Holden (i) ${ }^{2}$ \& Simon Heilbronner (I) 1,3,4凶

Gene tandem amplifications are thought to drive bacterial evolution, but they are transient in the absence of selection, making their investigation challenging. Here, we analyze genomic sequences of Staphylococcus aureus USA300 isolates from the same geographical area to identify variations in gene copy number, which we confirm by long-read sequencing. We find several hotspots of variation, including the csal cluster encoding lipoproteins known to be immunogenic. We also show that the csal locus expands and contracts during bacterial growth in vitro and during systemic infection of mice, and recombination creates rapid heterogeneity in initially clonal cultures. Furthermore, csa1 copy number variants differ in their immunostimulatory capacity, revealing a mechanism by which gene copy number variation can modulate the host immune response.

\footnotetext{
${ }^{1}$ Interfaculty Institute of Microbiology and Infection Medicine, Department of Infection Biology, University of Tübingen, Tübingen, Germany. ${ }^{2}$ School of Medicine, University of St Andrews, St Andrews KY16 9TF, UK. ${ }^{3}$ German Centre for Infection Research (DZIF), Partner Site Tübingen, Tübingen, Germany.

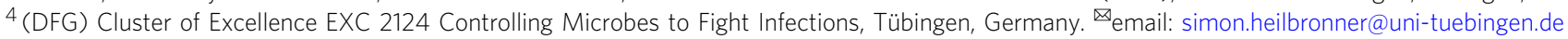


W ithin their natural environment, prokaryotes are constantly exposed to changing conditions ranging from shifting temperatures and changing nutrient availabilities to fluctuating levels of noxious compounds. The tremendous ability of prokaryotes to adapt to environmental changes is due to their capacity to alter their genetic material rapidly, which is a key element of their evolutionary success.

Prokaryotic genomes show a high degree of plasticity and acquisition of genetic traits by horizontal gene transfer (HGT) is well studied ${ }^{1}$. However, HGT relies on appropriate genetic material to be externally available and will be hampered if the bacterial community under selection is rather homogenous. Alternatively, genomic diversity created by single nucleotide polymorphisms (SNPs) can facilitate adaptation processes ${ }^{2}$. The same is true for genomic rearrangements that impact expression levels of genes ${ }^{3,4}$. Rearrangements occur in most cases stochastically by recombination between homologous DNA motifs and allow inversions as well as deletions or tandem amplifications of genetic material ${ }^{3}$. Tandem arrays of genes are most frequently caused by a RecA-dependent mechanism known as gene duplication and amplification (GDA). For the development of GDAs, the "accordion" model is well accepted and proposes that initial duplications can arise in RecA-dependent or independent fashions $^{5-7}$. After the primary duplication event, long perfect tandem repeats allow RecA-dependent amplification or, conversely, the loss of the duplication (segregation) at high rate ${ }^{6,7}$. Due to the high frequency of repetitive DNA segments in prokaryotic chromosomes gene copy number variants caused by GDAs create genetic and phenotypic heterogeneity in prokaryotic populations ${ }^{8-11}$. Selective pressures can favor certain copy number variants allowing stabilization of the arrays within the population. This phenomenon is often observed in the context of antibiotic resistance ${ }^{6,12}$. For instance, Nicoloff et al. have recently demonstrated that GDAs cause antibiotic resistant subpopulations in otherwise sensitive populations in many clinically relevant species $^{13}$. However, expansion and contraction of gene arrays should also harbor the potential to shape populations under unclear constrains such as pathogens or commensals facing a multitude of host-associated selective pressures. Therefore, the analysis of tandem amplifications in pathogens might pinpoint genetic loci under evolutionary pressure in the host. However, apart from loci encoding malfunctioning resistance determinants, it remains unclear whether special genomic regions are particularly prone to tandem amplification in the presence of environmental triggers such as antibiotic pressure or host immune defences.

The invasive pathogen Staphylococcus aureus is a major cause of healthcare and community-associated infections leading to severe morbidity and mortality. S. aureus shows a remarkable ability to adapt to the healthcare setting where strong artificial selective pressures such as antibiotics and disinfectants drive the evolution of pathogens to develop resistance ${ }^{14}$. In the age of next generation sequencing (NGS) thousands of genomes of strains from many different pathogenic species, including S. aureus, have been sequenced. This opens plentiful opportunities to identify gene copy number variations caused by GDAs between closely related strains and to link variation to phenotypic characteristics using experimental approaches.

Here, we test this approach using previously published NGS datasets of clinical populations of S. aureus USA300 from the urban area of New York city ${ }^{15}$. Our analysis reveals frequent gene copy number variations in loci that harbor repetitive sequences. Some of the proteins encoded at these loci have previously been linked to host colonization and virulence such as the surfaceanchored molecule SdrD and the Spl serine proteases. Most prominent is copy number variation within the lipoprotein gene array csa1. Using experimental approaches, we find amplification of $c s a 1$ and $s d r D$ to occur readily in vitro. The frequency of amplification is increased 10-fold when RecA is induced by the fluoroquinolone antibiotic ciprofloxacin, supporting the "accordion" model of amplification. csal copy number variants show distinct differences in Csal protein levels and altered immunostimulatory activity suggesting roles for the proteins in the interaction with the immune system. Using systemic models of invasive disease, we find that csal copy number variation also occurrs in vivo with a higher frequency than observed in any in vitro experiment. This depends on functional intact csal coding sequences with associated protein expression, suggesting that environmental constrains favor the creation of genotypic and phenotypic heterogeneity amongst clonal populations in vivo.

\section{Results}

Gene copy number variation is frequently observed in staphylococcal chromosomes. We thought to investigate whether gene copy number variation caused by GDAs in repetitive parts of the genomes creates unrecognized heterogeneity in $S$. aureus populations. In order to identify GDAs we focused on a published set of $S$. aureus USA300 genome sequences ${ }^{15}$ from New York that were obtained using Illumina HiSeq-technology which allows smooth coverage and accurate scaffolding. The short read datasets from 348 strains were mapped to the USA300 reference sequence FPR $3757^{16}$. Coverage across the chromosome was analyzed using a minimum window size of $100 \mathrm{bp}$ and areas showing $\geq 2 \times$ coverage were regarded as putatively amplified regions. We also included areas showing no coverage, which represent deletions. We focused on the core genome and the pathogenicity islands $\nu S a \alpha$ and $\nu S a \beta$ but excluded genes associated with other mobile genetic elements (MGEs) identified for USA300 (phages $\phi S A 2 u s a, \phi S A 3 u s a$, SCCmec, and transposases ${ }^{16}$ ) as differences in coverage of these will in part reflect similar MGEs inserted into various sites in the chromosome.

We found several areas of the core genome that varied in depth of coverage (Supplementary Data 1). These loci harbored highly repetitive DNA motifs, supporting the hypothesis that RecAdependent recombination might have created copy number variation. We discriminated three different types of repetitive elements facilitating recombination (Fig. 1a). Firstly, repetitive motifs were present as domains within several genes of a tandem array. The $s d r C D E$ locus encodes three cell wall-anchored proteins with highly repetitive serine-aspartate (SD) repeats $(85.9-88.3 \%$ identity between the genes). We identified 24 isolates lacking either $s d r D$ or $s d r E$ or both. All these deletions could be explained by recombination between the SD-encoding regions (Supplementary Fig. 1a, Supplementary Data 1). Secondly, repetitive domains were present within a single protein coding sequence (CDS). The surface-anchored protein SasG harbors highly repetitive G5-E domains ${ }^{17}$. The G5-E-encoding DNA was frequently overrepresented/deleted in individual isolates, suggesting that recombination altered the size of the open reading frame (Supplementary Fig. 1b, Supplementary Data 1). Finally, we realized that several $S$. aureus loci encode tandem arrays of genes that are highly similar over the entire length of the CDS. Amongst those was the array of serine proteases (splABCDEF) and the superantigen-like toxins (ssl) (Supplementary Data 1). However, most prominently associated with copy number variation in our set of isolates was the locus encoding surface displayed proteins previously named "conserved staphylococcal antigens 1 " (csal) ${ }^{18}$. The csal genes encode lipoproteins belonging to a group known as tandemlipoproteins (Lpps). Four loci encoding similar Lpps are present in the $S$. aureus chromosome (csal, the lipoprotein-like genes ( $l p l)$ encoded on the pathogenicity island $v \mathrm{Sa}^{19}{ }^{19}$, and two further loci here referred to as $l p p 3$ and $l p p 4$, respectively) (Supplementary 
a

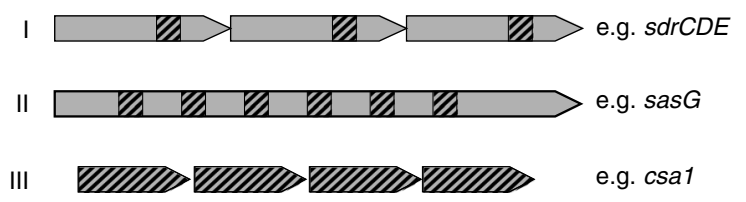

b

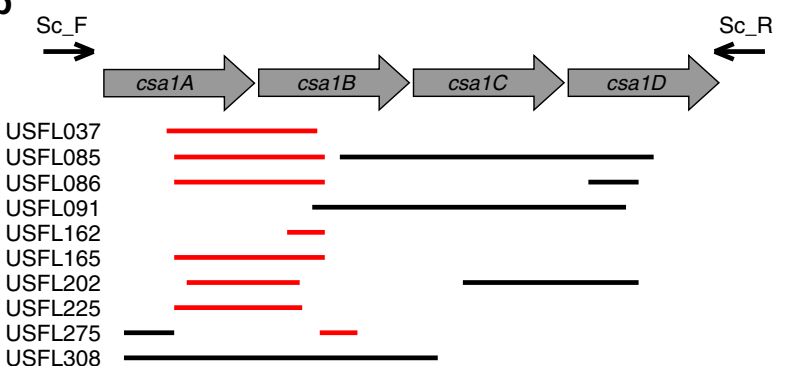

c

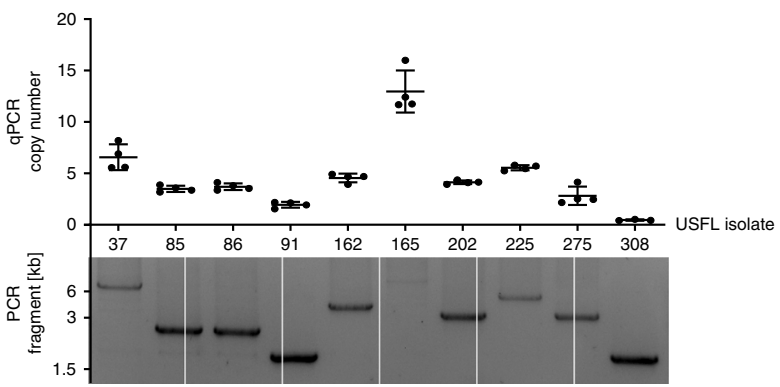

d

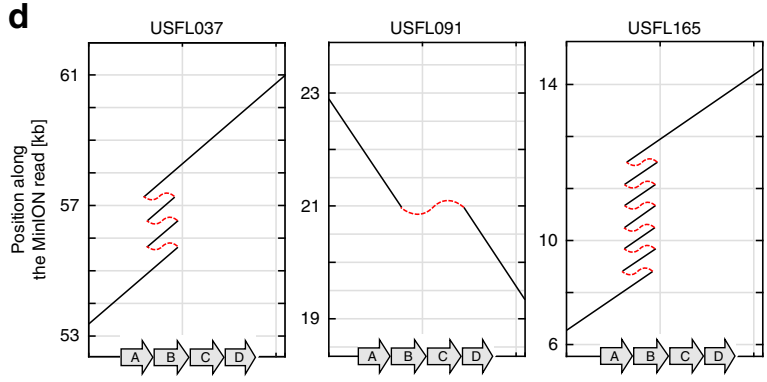

Fig. 1 csa1 gene copy number variation within the csa1 locus of S. aureus USA300 isolates. a Schematic diagram of the three categories of repetitive sequences creating copy number variation. Hatched areas denote regions of homology. $\mathbf{b}$ Schematic representation of the deletions and putative amplifications of csa1 in different isolates. Coding sequences are indicated. Red and gray lines represent increased coverage ( $>2$ fold) or deletions within the USFL isolates, respectively. c The upper panel shows the csa1 copy number of the indicated clinical isolates as measured by qPCR. Mean and SD of four replicate qPCRs on a single DNA isolation is shown. The lower panel shows DNA fragments amplified by conventional PCR using primers csa1_Sc.F and csa1_Sc.R indicated in (b). The experiment was performed once using the same batch of DNA used for qPCR. Source data are provided as a Source Data file. $\mathbf{d}$ USFL isolates were sequenced using MinION technology. For each sequenced isolate, a single long read that is aligned against the csa1 locus of USA300 FPR3757 is shown. Tandem amplifications manifest as multiple regions within the read with homology to csa1, allowing calculation of gene copy number. Hatched red lines emphasize that a single long read mapping repeatedly to the csa1 genes is analyzed. Genes csa1A, csa1B, csa1C, and csa1D are indicated. Shown are read_ch:51530_110 (USFL037); read_ch:10493_161 (USFL091); read_ch:40122_41 (USFL165). Source data are provided as a Source Data file.

Fig. 2). All lpp genes exhibit $46.1-81.9 \%$ identity. In the USA300 FPR3757 genome the $c s a 1, l p l, l p p 3$, and $l p p 4$ loci harbor four, ten, one, and three genes, respectively (Supplementary Fig. 2). Of note, despite the strong homology among all genes, two of them (lpp4C (SAUSA300_2424) and lpp3 (SAUSA300_0205)) do not encode lipoboxes, suggesting that the proteins are not anchored to the membrane ${ }^{20}$. Occasional deletions were observed in all loci in individual isolates but only csa1 showed putative amplifications in $80 \%$ of the clinical isolates (Fig. 1, Supplementary Data 1). The four csal genes of FPR3757 (csa1A-D) comprise 771, 771, 771, and $768 \mathrm{bp}$, respectively, and exhibit $61-80 \%$ identity (Supplementary Fig. 2). Each of the genes was occasionally deleted in individual isolates. In contrast, amplifications covered exclusively the csa1Acsa1B genes (Fig. 1b).

We assumed that the observed differences in NGS scaffolding indicated tandem amplification events within the array rather than additional copies of the genes on cryptic plasmids or on additional sites of the chromosome. To validate this, the $c s a 1 A B C D, s d r C D E$, and $s a s G$ loci of various isolates were amplified by PCR (Fig. 1c, lower panel, and Supplementary Fig. 1). Size differences between the amplified fragments were in agreement with predicted deletions and tandem amplifications. Of note, strain USFL165 harbored a csa1 locus that could not be amplified by PCR. We performed MinION long read sequencing of selected strains and extracted individual reads covering the csal array (Fig. 1d). This analysis confirmed tandem arrays of 7 , 3 , and 10 copies of $c s a 1$ genes in USFL037, USFL091, and USFL165, respectively. Of note, analysis of single reads identifies the gene copy number carried by an individual cell of the culture. However, since tandem arrays of genes are intrinsically unstable, individual cells might differ in copy number. Therefore, we designed qPCR primers to amplify a conserved fragment of all four csal genes simultaneously. Using chromosomal DNA as template, qPCR analysis allows us to determine the average gene copy number within the population. qPCR results correlated well with long read sequencing and identified $7 \pm 1,2 \pm 0.5$, and $13 \pm 2$ copies of csal genes in USFL037, USFL091, and USFL165, respectively (Fig. 1c, upper panel).

Amplification occurs constantly and the frequency is increased by antibiotic pressure. We sought to investigate the development of GDAs during growth of a single clone and chose the csa1ABCD locus as it showed frequent variation among the clinical isolates. We introduced a tetracycline $(\mathrm{Tc})$ resistance determinant (tetK) between $c s a 1 B$ and $c s a 1 C$ in USA300 LAC by allelic replacement. TetK is known to specify a resistance level that is gene-dosage dependent $^{21}$. The wild type was phenotypically sensitive to $2 \mu \mathrm{g} /$ $\mathrm{ml}$ Tc. The USA300 csa1::tetK strain was resistant to $2 \mu \mathrm{g} / \mathrm{ml} \mathrm{Tc}$ and displayed weak growth at Tc concentrations up to $10 \mu \mathrm{g} / \mathrm{ml}$ while growth was completely inhibited at concentrations exceeding $10 \mu \mathrm{g} / \mathrm{ml}$. We anticipated that spontaneous amplifications of csal would also span tet $K$ thereby increasing Tc resistance and providing a selectable phenotype. We grew USA300 csa1::tet $K$ over three consecutive days in six parallel broth cultures in the absence of Tc. Therefore, amplification within the broth culture was not favored by antibiotic selection, allowing estimation of stochastic amplification in the absence of selection. Each day, the cultures were plated on agar plates containing $20 \mu \mathrm{g} / \mathrm{ml}$ Tc and arising resistant colonies were picked to analyze the csal copy number by qPCR (Figs. 2 and 3). Not all Tc-resistant clones 
a
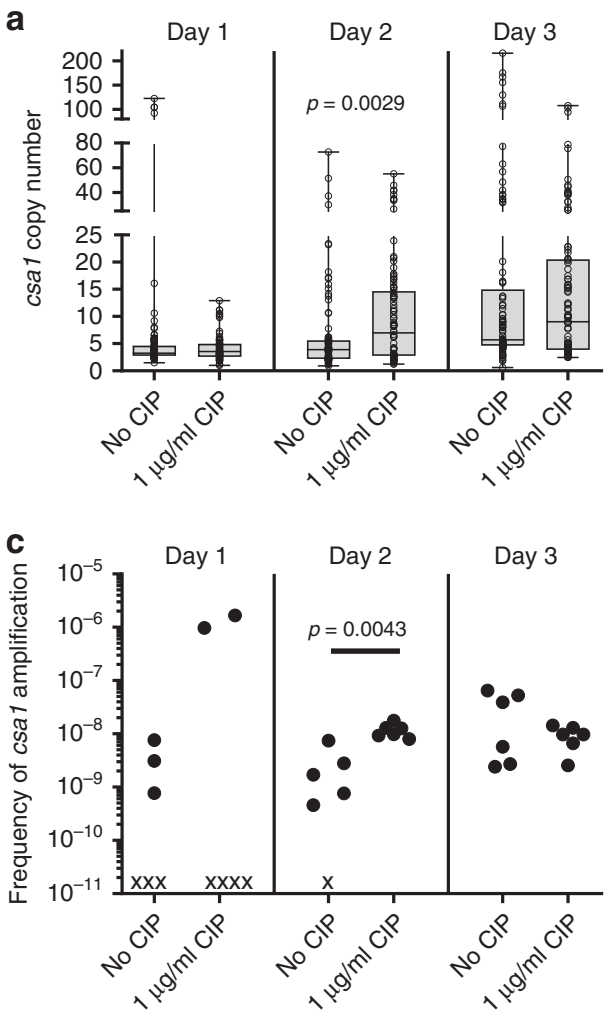

b

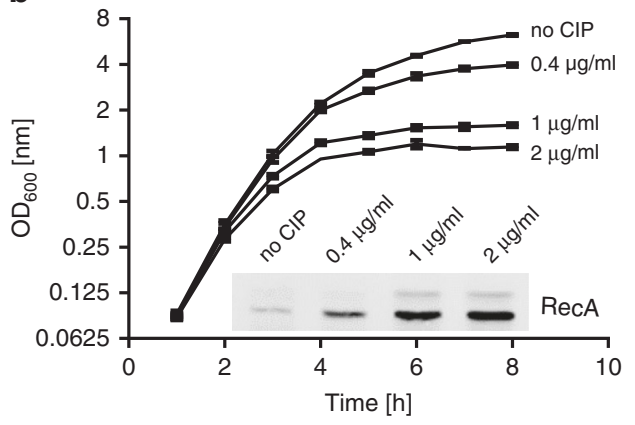

d

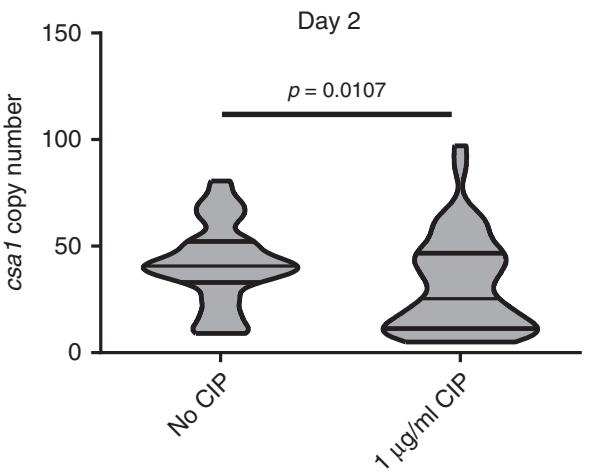

Fig. 2 Copy number diversification after in vitro evolution. a USA300 csa1::tetK was grown over three consecutive days in six parallel cultures in the presence or absence of ciprofloxacin (CIP). Each day, the copy number of up to 16 clones of each culture showing high Tc resistance was screened by qPCR. Upper and lower box limits and the horizontal lines within the boxes represent 25 and $75 \%$ percentiles and the medians, respectively. The whiskers of the plots indicate minimum and maximum range. All data points are shown. Data are derived from six independent experiments and represent: Day 1 , $n=96$ and $n=73$ of "no Cip" and "1 $\mu \mathrm{g} / \mathrm{ml} \mathrm{Cip",} \mathrm{respectively;} \mathrm{Day} \mathrm{2,} n=93$ and $n=96$ of "no Cip" and "1 $\mu \mathrm{g} / \mathrm{ml} \mathrm{Cip",} \mathrm{respectively;} \mathrm{Day} \mathrm{3,} n=93$ and $n=$ 96 of "no Cip" and "1 $\mu \mathrm{g} / \mathrm{ml} \mathrm{Cip",} \mathrm{respectively.} \mathrm{Datasets} \mathrm{were} \mathrm{not} \mathrm{normal} \mathrm{distributed} \mathrm{(D'Agostino} \mathrm{\&} \mathrm{Pearson} \mathrm{omnibus} \mathrm{test}<0.0001$ ) and statistical analysis was performed using two-tailed Mann-Whitney test. Source data are provided as a Source Data file. b Growth curves of USA300 csa1::tetK in TSB containing various concentrations of the antibiotic ciprofloxacin (CIP), which is known to enhance RecA expression. As expected, at increasing inhibitory concentrations of CIP, the growth rate of USA300 csa1::tetK decreased as assessed by measuring $\mathrm{OD}_{600}$ every $2 \mathrm{~h}$. This was accompanied by an increase in RecA protein levels as assessed in mid-exponential cells by Western blot analysis using LI-COR infrared technology. A representative blot is shown wherein the lower and upper bands represent RecA and an unspecific signal, respectively. Mean and SEM of three independent experiments are shown. Source data are provided as a Source Data file. c Frequency of amplification ( $T c_{20}$-resistant clones showing at least a 2 fold increase in csal copy number compared to the parental strain by qPCR) within the total population of living cells. Shown are the results of six independent experiments. $x$ indicates a culture in which amplification was not detected. Statistical analysis was performed using two-tailed Mann-Whitney test. Source data are provided as a Source Data file. d A USA300 csa1::tetK high copy number variant (harboring $~ 50$ copies of csa1) was grown over two consecutive days in three parallel cultures in the presence or absence of CIP. Each day, the copy number of the strains of 17 randomly chosen clones was screened by qPCR ( $n=51$ ). Shown is a violin plot, length of the box indicates minimum and maximum range. Width of the bar indicates accumulation of data points. Horizontal lines within the boxes represent 25 and $75 \%$ percentiles and the medians, respectively. Statistical analysis was performed using two-tailed Mann-Whitney test. Source data are provided as a Source Data file.

showed elevated csal copy numbers for reasons that were not apparent. Nevertheless, increased Tc resistance correlated frequently with an increased csal gene copy number and numbers as high as 100-200 copies were detected several times (Fig. 2a). After $24 \mathrm{~h}$ of growth, strains with amplifications in the csal locus were isolated in 2 of 6 parallel cultures. This number increased to $5 / 6$ and $6 / 6$ cultures after two and three days of growth, respectively. The copy numbers ranged from 4 to 200 csal genes with copy numbers as high as 100 detected at day 1 (Fig. 2a). Also, the level of copy number diversity increased over time with $I_{50}$-values (copy-number variation of $50 \%$ of the population surrounding the median) of 1.84 at day 1 and 3.24 and 10.36 at days 2 and 3, respectively (Fig. 2a).

To confirm tandem amplification of csal within such strains, we used the MinION technology to sequence four independently evolved isolates that displayed high copy number as measured by
qPCR (C6 - 149 \pm 22 copies; E28 - $78 \pm 10$; L38 - 64 \pm 3 ; III37 $26 \pm 2$ ) (Fig. 3a). From the MinION reads we extracted those that covered the csal gene array as shown in Fig. 3b. For strains C6 and E28 this approach produced individual reads $(70-120 \mathrm{~kb}$ in length) covering exclusively the csa 1 array but lacking upstream or downstream sequences, confirming the presence of tandem amplifications too large to be completely covered (Fig. 3b). This made precise copy number determination impossible but revealed that strains C6 and E28 harbored at least 77 and 72 copies, respectively. Analysis of strains L38 and III37 revealed single reads covering upstream and downstream sequences as well as the csa 1 array, suggesting 56 and 23 copies of the csa 1 gene. However, for both strains also reads lacking upstream and downstream sequences were identified suggesting that the sequenced populations were heterogeneous with respect to copy numbers of tandem amplifications and that some cells within the L38 and 
a

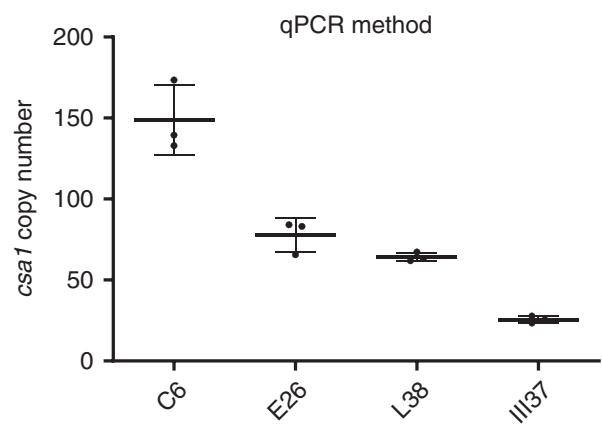

b
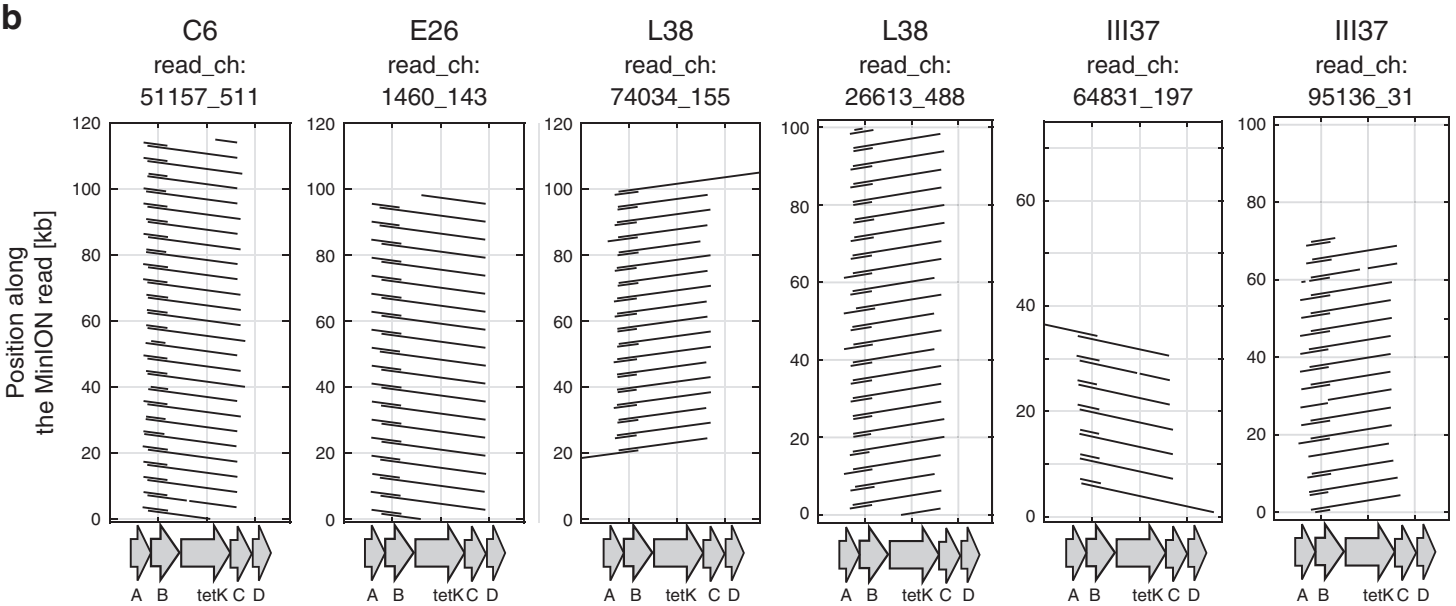

Fig. 3 Tandem amplification of csa1:tetK. a csa1 copy number of four independent, high Tc-resistant strains measured by qPCR. Mean and SD of three replicate qPCRs on a single DNA isolation is shown. Source data are provided as a Source Data file. $\mathbf{b}$ MinION sequence analysis of high Tc-resistant isolates. For each sequenced isolate, single reads covering the csa1 array were aligned against the csa1::tetK locus of the parental strain. Tandem amplifications manifest as multiple regions within the read with homology to csa1::tetK. Connecting lines within the read are omitted for reasons of clarity. Genes csa1A, csa1B, tetK, csa1C, and csa1D are indicated. Shown are read_ch:51157_511 (C6), read_ch:1460_143 (E28), read_ch:74034_155 (L38), and read_ch: 64813_197 (III37). Source data are provided as a Source Data file.

III37 isolates harbored at least 68 and 43 copies of csal genes, respectively (Fig. 3b).

Due to the high sequence similarity of the csal genes, we speculated that extension of the array is mediated by the SOS recombinase RecA. Fluoroquinolone antibiotics such as ciprofloxacin (CIP) are known to induce RecA expression ${ }^{22}$. Subinhibitory concentrations of CIP increased cellular RecA levels in a dose-dependent manner (Fig. 2b). This led to a more rapid diversification of the culture with differences compared to untreated cultures being most prominent at day two. This was reflected both in the level of diversity $\left(I_{50}\right.$ values of CIP-treated cultures increased from 2.401 at day 1 to 11.931 and 16.68 at day 2 and day 3, respectively) and in the frequency of strains harboring csa1 amplifications, which was $~ 10$ fold higher in CIPtreated compared to untreated cultures at day two (Fig. 2c). Only on day 3 the diversity of untreated cultures approached that of CIP-treated cultures.

Amplification and segregation events are two sides of the same coin. Amplification of gene arrays extends the length of sequence homology thereby increasing the frequency of recombination which leads to further diversification. To investigate the stability of the tandem arrays, we started an in vitro evolution experiment with a variant containing $\sim 50$ copies. After two consecutive passages in liquid broth the cultures were plated on agar without Tc to allow growth of all variants and the csal copy number of randomly chosen colonies was determined. The distribution of copy numbers varied significantly. The untreated culture displayed three distinct populations. $\sim 50 \%$ of isolates gathered tightly around the median csa1 copy number (40.7 copies) while additional populations harboring 52-81 copies and 9-32 copies were observed (Fig. 2d). In contrast, the distribution of copy numbers within the population of the CIP-treated cultures was more diverse. The median copy number was decreased to 25.3 but a clustering around the median was not observed. In contrast clustering of the population around the 25th percentile, which dropped from 32.96 to 11.34 upon CIP treatment, was observed. These results are in line with the "accordion"-model of GDAs leading to diversification of the population in high and low copy number variants with low copy numbers being favored under nonselective conditions. We speculated that our culture conditions were nonselective regarding the function of the Csal proteins and reflected stochastic creation of heterogeneity. To test this, we constructed the isogenic strain $\operatorname{csa} 1(\mathrm{FS}):: t e t K$. This strain carries a tet $K$-labeled csal locus where each gene is inactivated by a single nonsense mutation (Supplementary Fig. 3a). Passaging of this strain in the presence or absence of CIP revealed a similar pattern of amplification as observed for the functional locus, supporting the idea that Csa1 function was not selected for under our experimental conditions (Supplementary Fig. 3b).

Our bioinformatic analysis revealed frequent copy number variation also within the $s d r C D E$ locus in which recombination between SD repeat-encoding regions seemed to create frequent deletions. Again amplifications and segregations go hand in hand but in many cases deletions might be detected more conveniently as they represent the dead end of the GDA-mechanism due to their irreversible nature. Therefore, we hypothesized that $s d r C D E$ 


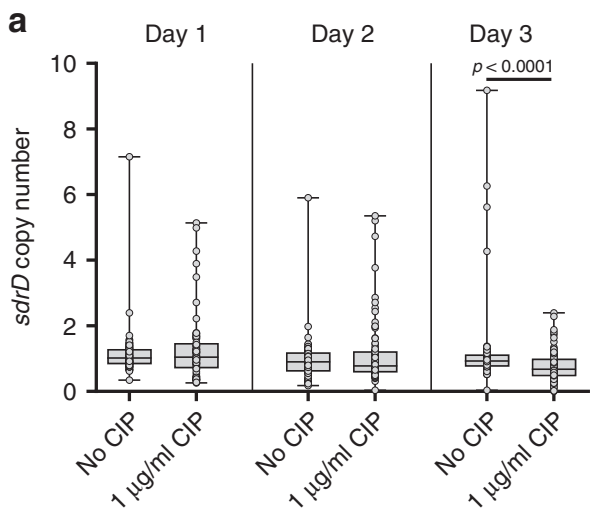

C

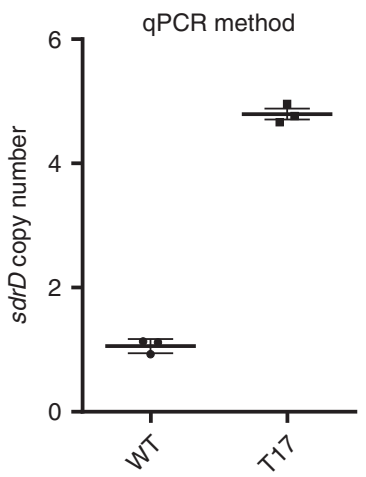

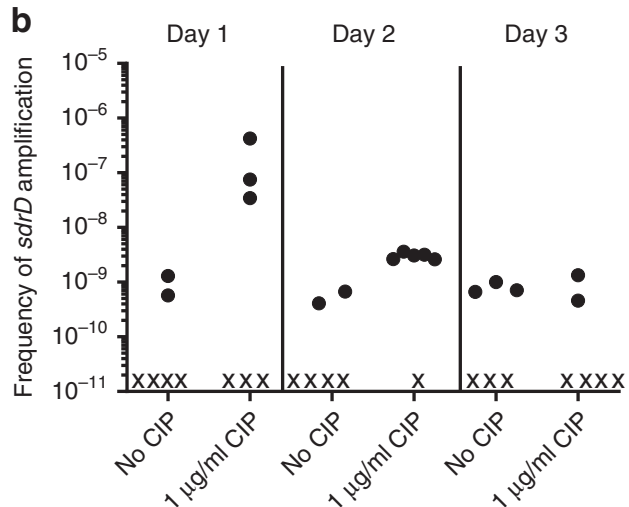

d
T17

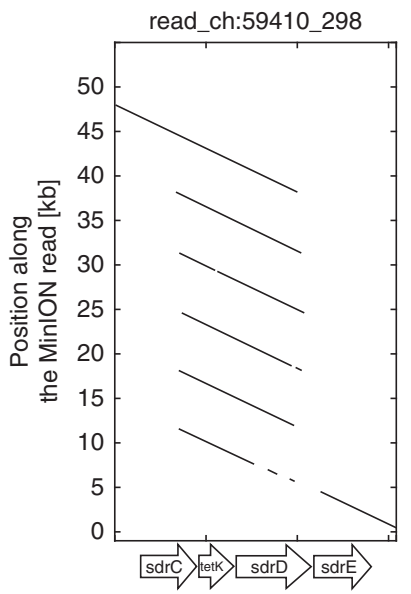

T17

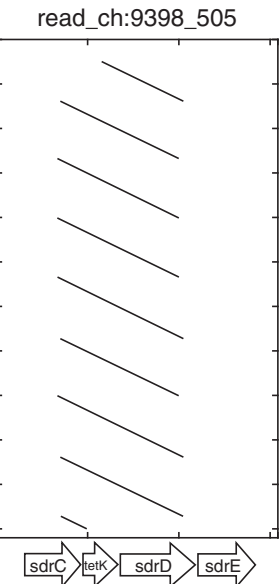

Fig. 4 Tandem amplification of sdrD::tetK. a USA300 sdrD::tetK was grown over three consecutive days in six parallel cultures in the presence or absence of ciprofloxacin (CIP). Each day, the copy number of up to 16 clones of each culture showing high Tc resistance was screened by qPCR. Upper and lower box limits and the horizontal lines within the boxes represent 25 and 75\% percentiles and the medians, respectively. The whiskers of the plots indicate minimum and maximum range. Data are derived from six independent experiments and represent: Day 1, $n=87$ and $n=76$ of "no Cip" and "1 $\mu \mathrm{g} / \mathrm{ml} \mathrm{Cip",}$ respectively; Day 2, $n=90$ and $n=95$ of "no Cip" and "1 $\mu \mathrm{g} / \mathrm{ml} \mathrm{Cip",} \mathrm{respectively;} \mathrm{Day} \mathrm{3,} n=78$ and $n=96$ of "no Cip" and "1 $\mu \mathrm{g} / \mathrm{ml}$ Cip", respectively. All data points are shown. Datasets were not normal distributed (D'Agostino \& Pearson omnibus test $<0.0001$ ) and statistical analysis was performed using two-tailed Mann-Whitney test. Source data are provided as a Source Data file. b Frequency of amplification ( $\mathrm{Tc}_{20}$-resistant clones showing at least a 2 fold increase in csa1 copy number compared to the parental strain by qPCR) within the total population of living cells. Shown are the results of six independent experiments. $x$ indicates a culture in which amplification was not detected. Source data are provided as a Source Data file. c sdrD copy number of a high Tcresistant strain in comparison to the parental strain measured by qPCR. Mean and SD of three replicate qPCRs on a single DNA isolation is shown. Source data are provided as a Source Data file. $\mathbf{d}$ MinION sequence analysis of the high Tc-resistant isolate. Single reads covering the $s d r C D E$ array were aligned against the sdrD::tetK locus of the parental strain. Tandem amplifications manifest as multiple regions within the read with homology to sdrD::tetK. Connecting lines within the read are omitted for reasons of clarity. Genes $s d r C$, tetK, sdrD and sdrE are indicated. Source data are provided as a Source Data file.

might also represent an expansible/contractible locus. We integrated the tetK cassette between $s d r D$ and $s d r E$ and performed similar experiments to those described above (Fig. $4 \mathrm{a}, \mathrm{b}$ ). In untreated cultures, strains harboring putative amplifications of $s d r D$ could be isolated from $2 / 6,2 / 6$, and $3 / 6$ parallel cultures at day one, day two, and day three, respectively. In contrast, when CIP was incorporated, $3 / 6,4 / 6$, and $2 / 6$ cultures harbored $s d r D$ copy number variants. Copy numbers ranged from 2 to 9 copies. Again, the highest copy number variation was apparent after 2 days in the presence of CIP. To confirm the copy number of $5 \pm$ 0.2 as quantified by qPCR for strain T17 (Fig. 4c), we performed MinION sequencing. This identified the presence of 6 tandem repeat copies but also revealed the presence of a heterogeneous T17 population, with some cells harboring $>8$ copies (Fig. $4 \mathrm{~d}$ ).

Altogether our results support the idea that the repetitive loci identified by the bioinformatic screen are subject to gene amplification processes and that the csal and the $s d r C D E$ loci represent expandable and contractible genetic loci. Stochastic events without selection of protein function create copy number variants and generate heterogeneity even within a clonal population.

Gene dosage correlates with protein expression levels. We detected csa 1 amplification levels reaching up to 200 gene copies suggesting that protein expression levels of these strains are increased. We created an isogenic $\Delta c s a 1$ deletion mutant lacking the entire csa1ABCD locus as a control and measured Csal protein amounts in membrane extracts of different copy number variants using LI-COR infrared Western blotting. The four proteins encoded in the csal locus are highly similar at the amino acid level. Mouse antiserum directed against Csa1C cross-reacted with the other Csal proteins and was used to detect all the Cas1 proteins simultaneously. The $\Delta c s a 1$ strain (0 copy number) had weakly immune-cross reactive proteins, most likely due to the 
a

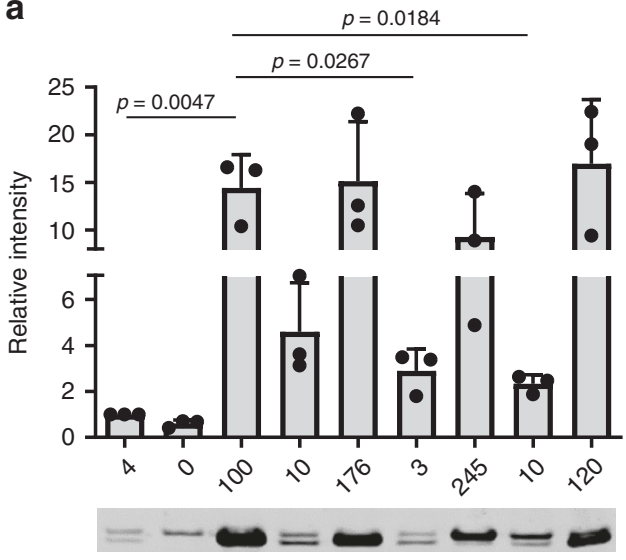

b

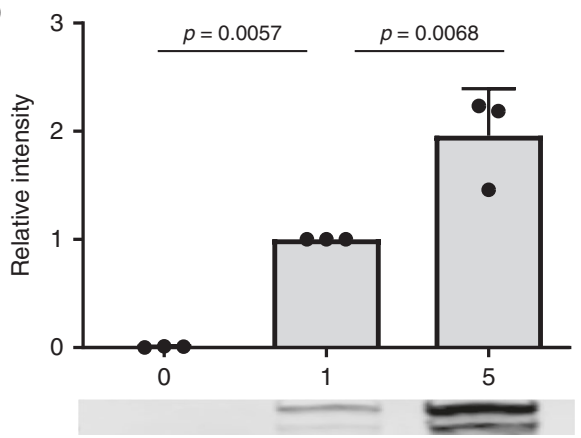

Fig. 5 Influence of copy number variation on protein expression levels.

a csa1 copy number variants were grown to stationary phase, cell membrane fractions were isolated and analyzed by SDS-page and Western blot. Csa1 proteins were detected using $\alpha$-Csa1 mouse serum, followed by goat $\alpha$-mouse secondary antibody coupled to IRDye800 (LI-COR). Protein amount within bands was quantified using LI-COR infrared technology. The fluorescent signal for the WT strain harboring four copies of csal was set to one in each experiment and the intensity of the copy number variants was expressed in relation to this value. Shown is a representative blot as well as the mean and SEM of three independent experiments. Statistical analysis was performed using one-way ANOVA $(F=9,434 ; D F=26)$ followed by Bonferroni's multiple comparisons test. Source data are provided as a Source Data file. b sdrD copy number variants were grown to stationary phase, cell wall-fractions were isolated and analyzed by SDS-page and Western blot analysis. SdrD was detected using $\alpha$-SdrD rabbit serum, followed by goat $\alpha$-rabbit secondary antibody coupled to IRDye800 (LICOR). Protein amount within bands was quantified using LI-COR infrared technology. The fluorescent signal for the WT strain harboring a single copy of $s d r D$ was set to 1 in each experiment and the intensity of the copy number variants was expressed in relation to this value. Shown is a representative blot as well as the mean and SEM of three independent experiments. Statistical analysis was performed using one-way ANOVA $(F=45,49 ; \mathrm{DF}=8)$ followed by Bonferroni's multiple comparisons test. Source data are provided as a Source Data file.

presence of common epitopes in lipoproteins encoded within the other three $l p p$ loci (Fig. 5a, Supplementary Fig. 2). However, compared to the WT strain harboring four copies, the signal intensity corresponding to Csal proteins increased in a genedosage dependent manner from $\sim 2$ to $\sim 20$ fold (Fig. 5a). This confirmed the gene dosage effect of csal amplification. However, copy numbers higher than 120 did not result in a further increase in protein expression levels (Fig. 5a).

Similarly, $s d r D$ copy number variants showed gene dosagedependent amounts of SdrD within the cell wall, with five gene copies increasing the amount of protein $\sim 2$ fold compared to a single gene copy (Fig. 5b).

Amplification of csa1 perturbs cytokine responses. Lipoproteins expressed by $S$. aureus are the most important Microbial Associated Molecular Patterns (MAMPs) ${ }^{23,24}$. They are shed from the bacterial cell surface in a surfactant-dependent manner ${ }^{25}$ and are recognized by toll-like receptor 2 (TLR2) on mammalian immune cells. The binding of lipoproteins to TLR2 activates a signaling cascade that culminates in the expression of cytokines and chemokines ${ }^{24}$. Therefore, we investigated whether csa1 amplification alters the immunostimulatory capacity of the bacterial supernatants, in which the Csa1 molecules are shed. We exposed TLR2 expressing Human Embryonic Kidney cells (HEK-hTLR2) to culture supernatants of csal copy number variants and found that levels of secreted IL-8 correlated with csal gene dosage (Fig. 6a). Interestingly, we observed spontaneous segregation events in several lineages, whereby one of the two independent cultures used for immunostimulation had undergone a drastic reduction in copy number (e.g. E26A and E26B in Fig. 6a). The culture supernatant showed an accordingly reduced immunostimulatory capacity. This confirmed that the observed phenotypes were caused by csal amplifications and were not due to secondary mutations. However, this phenomenon made replication of phenotypes for individual lineages difficult. Therefore, we grouped our samples according to the csal copy number measured on the day of the experiment. This showed that an increase from four to up to 30 copies had no detectable effects on HEKhTLR2 cells, whereas an increase from 90 to 200 copies enhanced IL-8 secretion by two- to threefold (Fig. 6b). In contrast, amplification of csal(FS) locus did not increase IL-8 secretion of HEKhTLR2 cells confirming that the observed phenotype was due to amplification induced Csal overexpression and not to cryptic secondary effects of the amplification (Supplementary Fig. 3c).

We hypothesized that the presence of four lpp loci in USA300 might mask the effects of csal copy number variation especially in variants harboring rather small amplifications (up to 30 copies). Therefore, we created a triple mutant deficient in $l p l, l p p 3$, and lpp4 followed by the isolation of csa 1 copy number variants. We stimulated the human macrophages cell line HL60 as well as primary human polymorphonuclear leukocytes (PMNs) with culture supernatants of csal copy number variants in the $\Delta l p l l p p 3 l p p 4$ background. Similar to HEK-hTLR2 cells, IL-8 secretion by HL60 cells and PMNs increased in a gene dosagedependent manner. Interestingly, these experiments also allowed detection of gene dosage effects associated with $\sim 30$ copies (Fig. 6c, d).

Gene copy number variation is created during infection. During invasive infection pathogens face hostile host factors that undermine bacterial cellular integrity, amongst these reactive oxygen species (ROS). As ROS are known to induce DNA damage 26,27 , we speculated that this might create traceable GDAs during proliferation within the organs of animals. To test this, we infected mice with a csa1::tetK low copy number variant and determined the csal number variation within this input culture as described above. Mice were sacrificed one day post infection and the $c s a 1$ copy number of highly Tc-resistant strains isolated from the kidneys of infected animals was determined (Fig. 7a). While copy numbers exceeding 8 copies were not detected in the cultures used for infection, we identified strains with $>8$ copies in 5 out of 6 infected mice. The distribution of copy numbers varied between mice, suggesting that heterogeneous populations arose independently in each mouse and generated population profiles unique to each animal. Interestingly, we found that the overall 

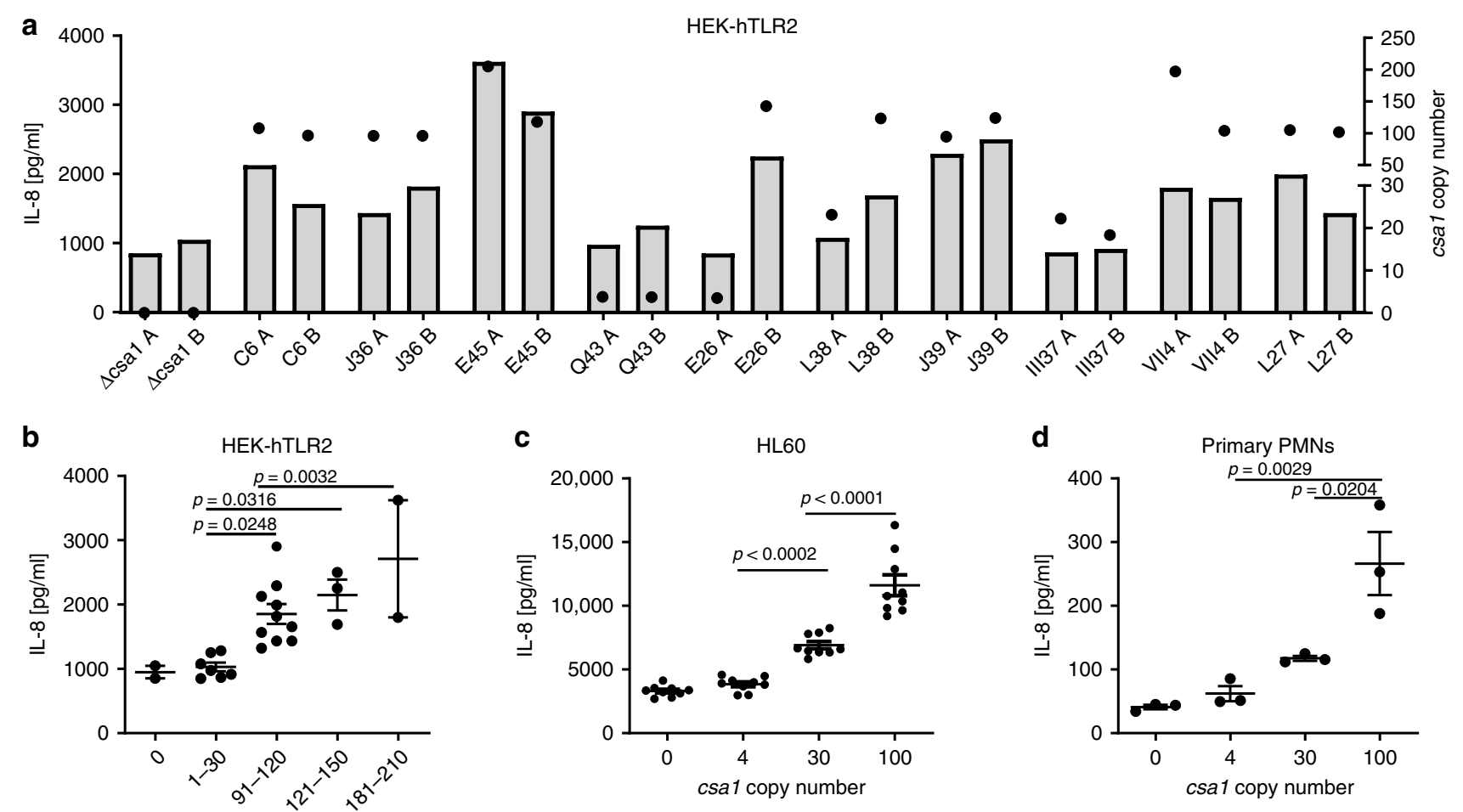

Fig. 6 Effects of csa1 amplification on the immunostimulatory capacity of strains. a Confluent lawns of HEK-hTLR2 cells were stimulated for $18 \mathrm{~h}$ with $0.5 \%$ culture filtrates of csa1 copy number variants grown to stationary phase. IL-8 protein levels within the supernatants were quantified by ELISA (R\&D Systems). IL-8 amounts are displayed as bars referring to the left $Y$-axis, data derived from a single stimulation experiment are shown. For each lineage, two parallel bacterial cultures (labeled with $A$ and B) were used for stimulation and the csal copy number of each independent culture determined by qPCR (displayed as filled circle referring to the right $Y$-axis). Source data are provided as a Source Data file. $\mathbf{b}$ IL-8 protein levels shown in (a) are expressed in function of the csa1 copy number of the stimulating strain. Sample sizes were the following: $0-n=2 ; 1-30-n=7 ; 91-121-n=10 ; 121-151-n=3 ; 181-210-$ $n=2)$. Mean and SEM is shown, statistical analysis was performed using one-way ANOVA $(F=7,710 ; D F=23)$ followed by Bonferroni's multiple comparison test. Source data are provided as a Source Data file. c $5 \times 10^{5} \mathrm{HL} 60$ cells were stimulated for $5 \mathrm{~h}$ with $1.5 \%$ culture filtrates of csa1 copy number variants ( $\Delta / \mathrm{p} / 1 / \mathrm{p} / 2 / \mathrm{p} / 3$ background) grown to stationary phase. IL-8 protein levels within the supernatants were quantified using ELISA (R\&D Systems). Data represent three independent supernatants of each copy number variant used in three independent stimulations ( $n=9$ in each group). Mean and SEM are shown. Statistical analysis was performed using one-way ANOVA $(F=3,632 ; D F=38)$ followed by Bonferroni's multiple comparison test. Source data are provided as a Source Data file. d Polymorphonuclear cells (PMNs) were isolated from fresh blood of healthy human volunteers. $5 \times 10^{5} \mathrm{PMNs}$ were stimulated for $5 \mathrm{~h}$ with $1.5 \%$ culture filtrates of csa1 copy number variants ( $\Delta / \mathrm{p} / \Delta / \mathrm{pp} 3 \Delta / \mathrm{pp} 4$ background) grown to stationary phase. IL-8 protein levels within the supernatants were quantified using by ELISA (R\&D Systems). Data represent three independent supernatants of each copy number variant used on PMNs of a single donor. Mean and SEM are shown. Statistical analysis was performed using one-way ANOVA $(F=15,74 ; D F=11)$ followed by Bonferroni's multiple comparison test. The experiment was repeated thrice with PMNs from different donors with similar results. Source data are provided as a Source Data file.

frequency of amplifications recovered from mice was $\sim 1000$ fold higher than the frequency of amplification recovered from any in vitro passaging experiment described above (Fig. $7 \mathrm{~d}$ ). These data suggest that the csal copy number variation created during infection might be favored by host selective pressures. To assess whether amplification of csal in vivo depended on functional protein expression or whether it represented stochastic, unselected variation created in the environment during infection, we repeated the infection experiment using the $S$. aureus strain carrying the inactivated csal locus (csal(FS)::tetK) (Fig. 7b, c). Interestingly, only very few high Tc-resistant clones were isolated from infected animals $24 \mathrm{~h}$ post infection and none of them displayed an increased csal(FS) copy number (Fig. 7b). Even when mice were sacrificed $48 \mathrm{~h}$ post infection and the csal(FS) copy number of highly Tc-resistant strains was analyzed, we did not detect extensive heterogeneity within the csal(FS)::tetK population. Indeed, only a single strain that was recovered from the kidneys of a single infected mouse carried an amplification (Fig. 7c). This strongly suggests that heterogeneity of the intact csa1 locus was selected in vivo by a yet undefined mechanism.
The increased immunostimulatory capacity of high copy number variants observed in cell culture led us to speculate that copy number variation might influence the severity of the disease caused by the strains. To test this, we infected mice either with a low copy number variant ( 4 copies) or a high copy number variant ( 100 copies) of csal genes and evaluated CFU counts within the organs of infected mice $72 \mathrm{~h}$ post infection (Fig. 7e). We did not observe an increased bacterial burden in mice infected with the high copy number variant, indicating that a high copy number of csal did not result in hypervirulence. As such the evolutionary benefit of csal amplification in vivo remains elusive.

\section{Discussion}

Recombination-mediated gene copy number variations are known to contribute significantly to the plasticity of prokaryotic genomes $^{3,10,11,28}$. Several studies have reported that gene amplifications can influence a variety of phenotypes ranging from antibiotic resistance ${ }^{12,13}$ (see ref. ${ }^{6}$ for an excellent review) to fitness advantages in the presence of unusual nutrients ${ }^{29-32}$. This suggests that this mechanism is highly relevant during adaption 
a
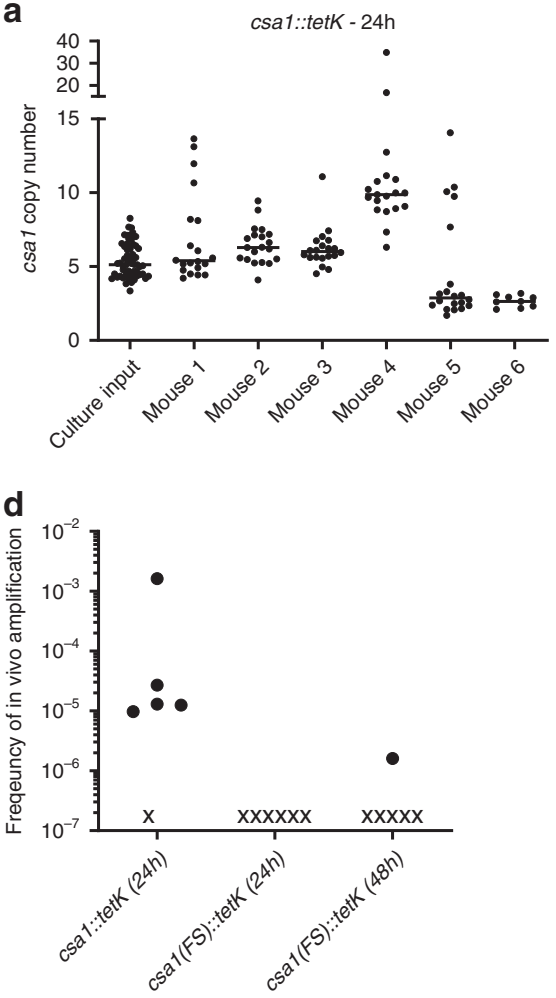

b

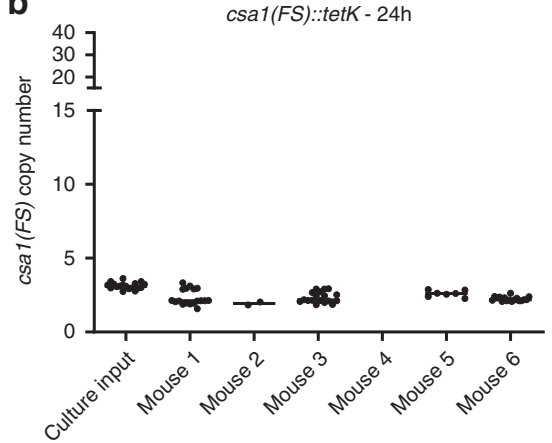

c

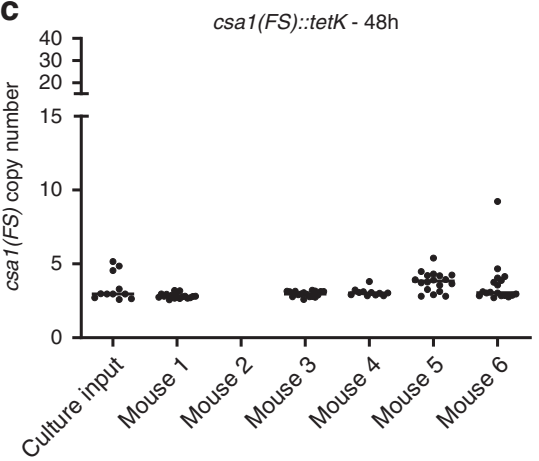

e

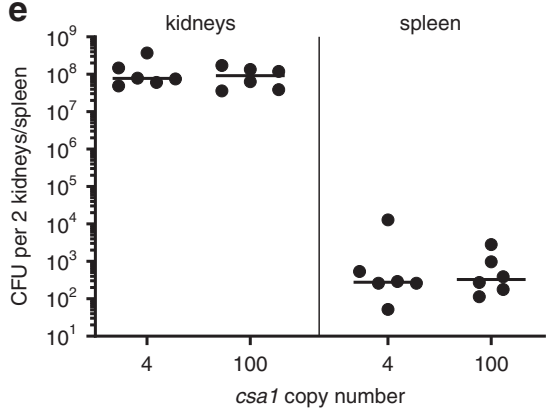

Fig. 7 Copy number diversification in vivo. a-c Six-week old female C57BL/6 mice were challenged with S. aureus live bacteria that carried either a functional csa1::tetK locus (a) or an inactivated csal(FS)::tetK locus (b, c). Mice were sacrificed $24 \mathrm{~h}$ or $48 \mathrm{~h}$ post infection as indicated. High Tc resistant clones arising from the input culture and from mouse kidneys were enumerated and csa1 (a) or csa1(FS) (b, c) gene copy number was determined. Horizontal lines show the median. Up to 19 arising clones were screened. No Tc 20 resistant clones were recovered from mouse 4 and mouse 2 in (b) and (c), respectively. Source data are provided as a Source Data file. $\mathbf{d}$ Frequency of amplification in mouse organs. qPCR analysis was used to calculate the frequency of amplification within each mouse (number of $\mathrm{Tc}_{20}$-resistant clones that showed at least a 2 fold increase in csa1 or csa1(FS) copy number compared to the parental strain within the total population of living cells recovered from each mouse) (dark-filled dots). $\mathrm{x}$ indicates a mouse in which amplification was not detected. Six mice were used in each group. Source data are provided as a Source Data file. e C57BL/ 6 mice were infected either with a low copy number variant ( 4 copies) or a high copy number variant ( 100 copies) of csal genes. Mice were sacrificed $72 \mathrm{~h}$ post infection and CFUs within the kidneys and spleen were enumerated. Horizontal lines show the median. Statistical analysis was performed using two-tailed Mann-Whitney test, but no significant differences were found. Six mice were used in each group. Source data are provided as a Source Data file.

to stressful environments. In line with this, gene amplification is known to be important for pathogens. Poxvirus host adaption is driven by gene amplification ${ }^{33}$. The eukaryotic pathogen Candida albicans develops amplifications within its host ${ }^{34}$ and the prokaryotes Hemophilus influenza and Vibrio cholerae can increase virulence by gene amplification ${ }^{35-39}$.

Accordingly, one can speculate that the analysis of copy number variations in populations under constraint may pinpoint regions under evolutionary selection, which might therefore be interesting targets for experimental investigation. However, even in the age of NGS, GDAs are rarely described in environmental or clinical isolates. There might be several reasons for this. Firstly, the intrinsic instability of gene amplification arrays might result in rapid segregation as soon as an isolate is removed from its natural habitat and any selective pressure stabilizing the GDA is lifted. During isolation and culturing a GDA might therefore be lost. Secondly, current NGS strategies and associated bioinformatic analysis make detection of GDAs difficult. Generally, the individual gene copies within a GDA array are identical making the detection of GDAs using short read sequencing techniques such as Illumina challenging. However, GDAs are indicated in NGS datasets by an increased scaffolding of reads ${ }^{40,41}$. NGS scaffolding is rarely uniform over the entire length of the chromosome. Additionally, increased coverage might also be caused by discrete additional copies of the gene on plasmids or on distant chromosomal locations. This makes secondary validation of presumed tandem amplifications essential. However, this is rarely within the scope of NGS projects. In our experiments copy number variation as suggested by scaffolding was confirmed by MinION sequencing demonstrating the potential of the approach.

GDAs in S. aureus isolates are poorly described and the known examples are only associated with increased resistance to antibiotics $^{42,43}$ or natural competence ${ }^{44}$. We speculated that gene copy number variations might be a prominent but neglected phenomenon. Indeed, we found the depth of NGS coverage to vary between closely related clinical $S$. aureus isolates, indicating certain genomic areas showing plasticity, especially in tandem arrays of genes with homology over the entire length of the CDSs such as csal, the serine protease encoding $s p l$ genes ${ }^{45}$ or the array of superantigen-like toxins (ssl) genes ${ }^{46}$. However, coverage variation of gene arrays possessing defined repetitive domains such as $s d r C D E$ or of single genes with repetitive motifs such as $s a s G$ was also observed. These clusters can be regarded as direct evidence of the model of "innovation, amplification and divergence" which suggests that GDAs increase gene dosage of genes under selection. Divergence of gene copies by mutation creates functional diversity which is subsequently retained ${ }^{47-49}$. Along this line, the repetitive arrays of $S$. aureus are generally thought to encode functionally distinct proteins that are stabilized and retained within the population ${ }^{50,51}$. However, it has been 
described that the number of repeats within these arrays can differ among $S$. aureus isolates from different lineages ${ }^{52,53}$, suggesting ongoing diversification. Our analysis shows that even closely related clinical isolates can differ within the number of repeats and our experimental approaches show that copy number variation is created during bacterial growth in vitro and in vivo. This indicates that the loci are not as stable as generally anticipated and might suggest a long-lasting selective pressure acting on these arrays.

The scaffolding analysis identified a number of core genome loci that are frequently associated with deletions but not amplifications (e.g. $s d r C D E$ ). This finding can be explained by RecApromoted recombination between sister chromatids during chromosome replication. One consequence of such recombination is one daughter cell with a duplication and another with a deletion of the DNA fragment. Duplications will be intrinsically unstable in following generations while deletions will be fixed in the progeny even if they are not beneficial to the lineage. We speculate that the loci that show frequent deletions in the collection of clinical isolates might undergo GDAs but amplifications might be lost during isolation and cultivation of the strains.

We investigated the plasticity of $c s a 1$ and $s d r C D E$ arrays in changing environments and found both loci to expand and contract, creating heterogeneous populations. Amplification in vitro was independent of protein function suggesting that stochastic recombination events drove the creation of gene copy number variation. The gyrase inhibitor ciprofloxacin, which causes DNA damage and stimulates the SOS response and RecA activity, increased diversification, supporting the idea of RecAmediated recombination at the origin of the observed heterogeneity. Strikingly, bacterial populations recovered from infected animals exhibited csal amplification only when the locus was intact. In vivo amplification of the inactivated csal(FS) locus was not observed, suggesting that the in vivo function of the Csal confers a selective advantage and might impact host-pathogen interaction. In addition, the intact csal locus amplified with a $\sim 1000$-fold increased frequency in vivo compared to any in vitro experiment. However, these datasets need to be compared with care. The bacterial generation times in vivo are unclear and bottlenecks will stochastically drive population structure during infection ${ }^{54}$. Nevertheless, our data suggest that heterogeneity is created in vivo and its frequency most likely enhanced by host immune pressures such as reactive oxygen species damaging the bacterial nucleic acids ${ }^{55}$.

The biological roles of csal are unclear and we therefore investigated whether amplification of the array affects immune relevant phenotypes. Bacterial lipoproteins are ligands of Toll-like Receptor 2, which recognizes diacyl and triacyl lipoproteins in combination with TLR1 and TLR6, respectively ${ }^{56}$. Interestingly, $S$. aureus USA300 possesses four clusters encoding Lpps (Supplementary Fig. 2), all of which are located close to the origin of replication resulting in an increase in average gene copy number due to bidirectional replication of the chromosome. The locus with the highest $l p p$ copy number ( $l p l-10$ genes in USA300 LAC) is located within the pathogenicity island vSaa and is important for virulence ${ }^{19,24,57,58}$. It was suggested that the immunostimulatory effects observed in vitro are outbalanced by the biological activities of the proteins ${ }^{19}$. Similar, it was shown that the expression of the lpp4 cluster is upregulated by $\beta$-lactam antibiotics resulting in increased immune stimulation and pathogenicity ${ }^{59}$. Therefore, we assumed that csal amplification might perturb the immune response. We found that HEK-hTLR2 cells as well as HL60 macrophages and primary human PMNs reacted with a gene dosage-dependent increase in IL-8 secretion when stimulated with supernatants of csal copy number variants. Quantitative differences in the expression and release of TLR2 ligands have been shown to be crucial during S. aureus pathogenesis as they allow modulating host immune responses ${ }^{25,60}$. Highly invasive strains such as USA300 do frequently show increased TLR2 activation ${ }^{60}$. However, strains harboring $\sim 100$ copies of cas1 were not hypervirulent in our infection experiments, making the benefit of in vivo csal amplification ambiguous. In addition, only moderate copy number of 8-30 copies were detected in organs of infected mice. It can therefore be speculated that gene dosage-dependent expression levels might be under constant selection with benefits of amplification being outweighed by disadvantages at a certain level of amplification.

Csal proteins are known to be immunogenic and were proposed as vaccine candidates ${ }^{18}$. Additionally, recombination between $l p p$ genes has been proposed to cause phase variation by creating chimeric surface-located proteins ${ }^{52}$. Recombination and amplification of csal might therefore prevent recognition by antibodies. However, further experiments are needed to confirm this hypothesis.

We also found the $s d r C D E$ locus to be expandable and contractible. This locus seems indeed to fulfill all criteria for the "Innovation, amplification and divergence" model. The DNA encoding the C-terminal SD-stalk in $s d r C, s d r D, s d r E$ is highly similar. In contrast the regions encoding the $\mathrm{N}$-terminal ligandbinding domains of these cell wall-anchored proteins are divergent. SdrC binds neurexin ${ }^{61}$. SdrD binds desmoglein $10^{62}$ and facilitates adherence to desquamated nasal epithelial cells ${ }^{63}$. SdrE (Bbp) binds complement factor $\mathrm{H}^{64}$ and bone sialoprotein ${ }^{64}$. Our observations support the idea that the genes were created by ancient GDA and mutations allowed functional diversification, in this case facilitating recognition of different host molecules. Our results indicate that this mechanism is still acting on this array. We did not observe amplification of $s d r D$ to improve adherence to immobilized desmoglein 10. Therefore, it is tempting to speculate that the SdrCDE-encoded proteins mediate weak adherence to yet unknown ligands and that these interactions can be strengthened by increasing protein expression. However, this hypothesis remains challenging to explore. Most likely positive selection of amplifications will only occur during colonization/ infection of human body sites that provide "unusual" ligands for SdrCDE. As such, only sampling strategies that strictly separate the different sites of colonization/infection, followed by NGSscaffolding analysis, will allow a better understanding of the host factors that select for high $s d r C D E$ copy number variants. The relevance of such strategies is exemplified by the work of Waller et al. which reported the frequency of gene copy number variation in Streptococcus equi during host adaption ${ }^{41}$.

Finally, we also discovered that the size of repeats within an individual gene can vary significantly between closely related strains. This was observed for the cell-wall-anchored protein SasG. Such recombination harbors a risk of disrupting the open reading frame by introducing frame shift mutations. However, our analysis did not identify such mutations and we did detect SasG of different molecular weight in cell wall fractions of G5-E copy number variants. Interestingly, it has been observed before that the size of SasG varies among S. aureus lineages. It was also shown that very large variants of SasG promote biofilm formation by mediating $\mathrm{Zn}$-dependent, intercellular interactions ${ }^{53}$. At the same time adherence to several host matrix molecules was reduced by long SasG variants by preventing other cell wallanchored proteins to bind their ligands ${ }^{65}$. It is tempting to speculate that accordion like-expansions and contractions of SasG promote phenotype switching allowing a heterogeneous population to colonize different body surfaces.

Our experiments show that gene copy number variations are omnipresent in staphylococcal populations and can be detected by NGS analysis. Expansions and contractions of gene arrays 
Table 1 Bacterial strains used in this study.

\begin{tabular}{|c|c|c|}
\hline Strains & Genotype & Source \\
\hline S. aureus USA300 LAC & Wild type & 71 \\
\hline S. aureus USA300 cas7::tetK & tet $K$ inserted into the csal locus & This study \\
\hline S. aureus USA300 cas1(FS)::tetK & tetK inserted into the inactivated csal locus & This study \\
\hline S. aureus USA300 $\Delta$ cas1 & Clean deletion of the $c s a 1 A B C D$ locus & This study \\
\hline S. aureus USA300 $\Delta s d r D$ & $\begin{array}{l}\text { Transposon insertion mutant derived from the Nebraska } \\
\text { mutant library ID NE1289 }\end{array}$ & $\begin{array}{l}\text { https://www.unmc.edu/pathology/csr/ } \\
\text { research/library.html }\end{array}$ \\
\hline S. aureus USA300 $\Delta / p / \Delta / p p 3 \Delta / p p 4 \Delta$ cas1 & Clean deletion of all 4 lpp loci, & This study \\
\hline S. aureus USA300: $\Delta / p / \Delta / p p 3 \Delta / p p 4$ cas7::tetK & $\begin{array}{l}\text { Clean deletion of } 3 \mathrm{Ipp} \text { loci as tetK inserted into the } \\
\text { csa1 locus }\end{array}$ & This study \\
\hline USFL037 & Clinical MRSA isolate & 15 \\
\hline USFL085 & Clinical MRSA isolate & 15 \\
\hline USFL086 & Clinical MRSA isolate & 15 \\
\hline USFL091 & Clinical MRSA isolate & 15 \\
\hline USFL118 & Clinical MRSA isolate & 15 \\
\hline USFL162 & Clinical MRSA isolate & 15 \\
\hline USFL165 & Clinical MRSA isolate & 15 \\
\hline USFL190 & Clinical MRSA isolate & 15 \\
\hline USFL2O2 & Clinical MRSA isolate & 15 \\
\hline USFL225 & Clinical MRSA isolate & 15 \\
\hline USFL234 & Clinical MRSA isolate & 15 \\
\hline USFL275 & Clinical MRSA isolate & 15 \\
\hline USFL308 & Clinical MRSA isolate & 15 \\
\hline USFL311 & Clinical MRSA isolate & 15 \\
\hline
\end{tabular}

occurred readily in vitro and in vivo, created heterogeneous populations and copy number variants differed in clinically relevant phenotypes. This study suggests that scaffolding analysis of NGS datasets can help identifying genomic areas under evolutionary constraint. It also suggests that scaffolding analysis of strains isolated during and after infection might pinpoint bacterial genes associated with host-adaptation, virulence or antibiotic resistance, thereby increasing the heuristic value of NGS analysis.

\section{Methods}

Chemicals. If not stated otherwise, all reagents were obtained from Sigma Aldrich.

Bioinformatic analysis of NGS data. Whole genome sequence (WGS) data from the study by Uhlemann et al. ${ }^{15}$ were used to look for copy number variants in the genomes of USA300 isolates. Paired-end reads were mapped against the core chromosome of the ST8 USA300 reference genome sequence FPR3757 (accession number CP000255) ${ }^{16}$ using SMALT (www.sanger.ac.uk/science/tools/smalt-0) ${ }^{41}$. To identify amplifications and deletions, read coverage along the reference genome was examined using a continuous hidden Markov model with three states: $0 \times$ coverage, $1 \times$ coverage, and $\geq 2 \times$ coverage. Initial and transition frequencies were fitted to the data using a Baum-Welch optimization, and the most likely sequence of hidden states was calculated using the Viterbi algorithm ${ }^{66}$.

Bacterial strains and growth conditions. All bacterial strains are listed in Table 1. If not specified otherwise, $S$. aureus strains were grown at $37{ }^{\circ} \mathrm{C}$ in Tryptic Soy Broth (TSB) or on Tryptic Soy Agar (TSA) (Oxoid) with shaking at $160 \mathrm{rpm}$ for liquid cultures. E. coli strains were grown at $37^{\circ} \mathrm{C}$ either in Lysogeny Broth (LB) or on Lysogeny Agar (LA) (Oxoid) with shaking at $160 \mathrm{rpm}$ for liquid cultures.

Determining copy number via MinION sequencing. Genomic DNA was sequenced on an Oxford Nanopore Technologies MinION system (NCCT, Tübingen) with $5 \mathrm{k}$-fold mean coverage and a $10 \mathrm{~kb}$ mean read length. For each sample, individual raw reads containing either tet $K$, the region leading into the duplicated region ( $500 \mathrm{bp}$ before and of the first gene in the operon), or the region following the duplicated region (500 bp of and after the last gene in the operon) were identified using NUCmer (MUMmer v3.2367 https://anaconda.org/bioconda/ mummer), run using Bioconda v4.8.068 (https://bioconda.github.io/). Optional arguments were used to minimize erroneous matches and allow for multiple mappings (nucmer -c 200 -maxmatch). For efficiency, only reads of at least $30 \mathrm{~kb}$ in length were considered. Reads identified as covering the copy region were mapped onto the entire operon ( $c s a 1$ or $s d r$ ) to determine gene copy numbers (nucmer -c 200, show-coords).
Plasmid construction. A list of plasmids used in this study is available in Table 2. The short intergenic region between $\operatorname{csa} 1 B$ and $c s a 1 C$ was chosen for insertion of tet $K$. A fragment containing $500 \mathrm{bp}$ upstream and 500 bp downstream was synthesized (Eurofins). In this process a HindIII restriction site was introduced as well as a $5^{\prime}$ SacI site and a $3^{\prime}$ SalI site between $\operatorname{csalB}$ and $c s a 1 C$. The recombinant fragment was cloned blunt end into pBluescript. The resulting pBluescript:csa was linearized using HindIII. tet $K$ was amplified from pT181 with HindIII sites at either end and cloned into pBluescript:csa. The resulting cassette (csa1B-tetK-csalC) was excised from pBluescript and cloned into the thermosensitive plasmid pIMAY. For deletion of $c s a 1, l p l, l p p 3$, and $l p p 4,500$ bp DNA fragments upstream and downstream of the genes to be deleted were amplified by PCR. A sequence overlap was integrated into the fragments to allow fusion and creating an ATG-TAA scar in the mutant allele. The $1 \mathrm{~kb}$ deletion fragments were created using spliced extension overlap PCR and cloned into pIMAY. All the oligonucleotides are summarized in Table 3.

For creation of the inactivated csal locus (csal(FS)) a recombinant locus was synthesized (Genewiz). The recombinant locus contains four point mutations that create ochre nonsense codons in triplet 3 of $\operatorname{csalA}, \operatorname{csa} 1 \mathrm{~B}$, and $\operatorname{csa} \mathrm{lC}$ as well as an opal nonsense codon in triplet 3 of $c s a 1 D$. tet $K$ was inserted into the array as described above and the csal(FS)::tetK fragment was cloned into pIMAY. The fragment was integrated into USA $300 \Delta c s a 1$ by allelic exchange ${ }^{69}$.

Quantitative PCR to determine the csa1 and sdrD copy number. S. aureus chromosomal DNA was isolated using the BioEdge chromosomal DNA isolation kit and Quick-DNA 96 Plus Kit according to the manufacturer's recommendation with an additional incubation with $1 \mu \mathrm{g} / \mathrm{ml}$ lysostaphin for $1 \mathrm{~h}$ after resuspension. Chromosomal DNA was adjusted to $100 \mathrm{ng} / \mu \mathrm{l}$ and a 1:10 serial dilution was used to create standard curves. qPCR primers were designed using the Primer3 software and are listed in Table 3. Primers directed against the origin of replication (ori) were used as the single copy reference. Primers against csal were directed against a highly conserved stretch of the coding sequence to allow amplification of a fragment from all four genes $(\operatorname{csa} 1 A / B / C / D)$. Primer binding sites were only partly conserved in the other $l p p$-coding sequences. No amplification was detected using chromosomal DNA of the $\Delta c s a 1$ strain, ruling out amplification of $l p l, l p p 3$ and lpp4 genes. Primers against $s d r D$ amplified a 5 , fragment of $s d r D$. The relative abundance of $c s a 1$ and $s d r D$ in relation to ori was calculated using standard curves. qPCR was performed using the Quantsudio3 (Applied Biosystems) and the "SYBR Green Mastermix" (Applied Biosystems).

Bacterial growth to isolate copy number variants. S. aureus USA300 csa1::tetK was used to inoculate $20 \mathrm{ml}$ TSB and incubated for $6 \mathrm{~h}$ at $37^{\circ} \mathrm{C}$. Cells were harvested, washed and used to inoculate $20 \mathrm{ml}$ TSB at an $\mathrm{OD}_{600}=0.05$. When indicated, ciprofloxacin (Fluka) was added to the culture. Cultures were incubated for $24 \mathrm{~h}$ at $37^{\circ} \mathrm{C}$. The next day bacteria were diluted (1:20) in fresh TSB and incubated for additional $24 \mathrm{~h}$ at $37^{\circ} \mathrm{C}$. After incubation serial dilutions were prepared and CFUs on TSA (total counts) and on TSA containing $20 \mu \mathrm{g} / \mathrm{ml}$ tetracycline (TSA $_{\mathrm{Tc2} 2}$, putative amplifications) were enumerated. The csal copy number of 
Table 2 Plasmids used in this study.

Plasmids

\section{pT181}

PIMAY

pIMAY:csa1::tetK

pIMAY:csa1(FS)::tetK

pIMAY: $\Delta c s a 1$

pIMAY: $\Delta / p l$

pIMAY: $\Delta / p p 3$

pIMAY: $\triangle / p p 4$

\section{Description}

Source

Staphylococcal plasmid encoding tetK

Thermosensitive vector for allelic exchange

Fragment for insertion of tetK into the csa1 locus of USA300 LAC

Fragment for intergration of the inactivated csa1-tetK locus into USA300 $\Delta$ csa1.

Fragment for deletion of the csa1 locus of USA300 LAC

Fragment for deletion of the Ipl locus of USA300 LAC

Fragment for deletion of the Ipp3 locus of USA300 LAC

Fragment for deletion of the Ipp4 locus of USA300 LAC

Table 3 Oligonucleotides used in this study.

\section{Name}

csa1_Sc.F

csa1_Sc.R

qCsa1_F

qCsa1_F

qOri_F

qOri_R

qSdrD_F

qSdrD_R

sdr_Sc.F

sdr_Sc.R

0293_Sc.F

0296_Sc.R

csa1KO_A

csa1KO_B

csa1KO_C

csa1KO_D

csa1_ScF

csal_ScR

IpIKO_A

IpIKO_B

Ip1KO_C

IpIKO_D

Ipl_ScF

Ipl_ScR

Ipp3KO_A

Ipp3KO_B

Ipp3KO_C

Ipp3KO_D

Ipp3_ScF

Ipp3_ScR

Ipp4KO_A

Ipp4KO_B

Ipp4KO_C

Ipp4KO_D

Ipp4_ScF

Ipp4_ScR

Sdr_tetK_A

Sdr_tetK_B

Sdr_tetK_C

Sdr_tetK_D

Sdr_tetK_Scr.F

Sdr_tetK_Scr.R CAACTTTATTTCCAGTGGTAGATTGTACAC

tetK_F

tetk_R

5'-3' sequence

GATATTAAGACGAGTATGAAAATAGTTAG

ATTTTACAGCAACATATTTGAATTTC

TCCAGAGGTGCCGAGTTATT

TTTATATCCAACTGATGAGCCTTTT

TCGTGATAACGAAGGTGAAGC

GGTGGTCGATCACTCGAAAT

GCGACAACTTCAGCAAGTGA

TGGTGAAGCTTGCTCATCTG

GAGCAATGTTATTAATTAAAATAAGATG

GAATAAGGATTCCATTTAACATATACAC

GGAAATAAGTGTAGAGAATAAATTAATAG

TATTATTATTTGATGACAACTTTATGG

CATCTTACAACTCTCTTCTTTTTAAAATG

CATTTGAAACGAAAATTAATAATGG

CTTTGGTTCGAATGATATGTACGC

AAGTATGATCTTAAGTTGTCTTTTGTAGC

TAGAAATAGGAGCTGGATTATAAACC

CATACATTCCCACCGTTTCTCAAAATAC

TATGTATTTGTAACGCCTATGTGGAACC

CGATGGATGCATGACAAATATTGGG

CATTTCACATCCCCATTTTTATTTTTG

TAAAATGGTTTACTAAATCTAATAGAAC

TTATATAAACTCTCTCGTCTCTCTCTA

ACTCGAGCTCAACCAATGAGTACGG

ATCGTAAAACGGGATCCAACATTTGTGT

CATTTGTCGACGTTTCATTACCTTGAGA

GATAGCGATTCAGATTCAGATGCAG
AGGGAACAAAAGCTGGGTACCACTATGATAAAAAAGTTGAAG

GAAGAGAGTTGTAAGATGTAATCATCCACACACACGATTC

ATAGGGCGAATTGGAGCTCCATAAGCAACTGAATCACAAG

CACTAAAGGGAACAAAAGCTGGGTACCCAATATAACTTAATTCATGTTCTAAG

CATATAAATAATTAATTATTTTGTATATTTCTC

CAAAATAATTAATTATTTATATGTAGGAAGTATAAAATAGATTTAAAAG

ACTCACTATAGGGCGAATTGGAGCTCTAGAACTACCGCATCTCTTCCACCTA

CACTAAAGGGAACAAAAGCTGGGTACCATATTGATGCTATTTCAATTGCAGG

GTATTTTGAGAAACGGTGGGAATGTATGTAATACTTATGCTGTAATTATAGAAAC

CGACTCACTATAGGGCGAATTGGAGCTCGAAGTTAGTGCACATATTGAAGATTTAAG

CACTAAAGGGAACAAAAGCTGGGTACCGGAATTAAAATGTATATTTTTGTACAG

AAATAAAAATGGGGATGTGAAATGTGAATATCAAATAAAACCTGGTAATA

CGACTCACTATAGGGCGAATTGGAGCTCTACAAGTCTAATATTACATGAATTTCC

GGATCCCGTTTTACGATAAAGAAAAATAATTAAAGTATTG

GTCAACGGGGTTTTCAATGGGGAAAGCTTCACAGAA

CATAACACTAACAAAACATCGCTGTTAAAGCTTTTTTATTAC purpose

Screening for length variation in the csal locus.

Screening for length variation in the csal locus.

qPCR of the csal locus.

qPCR of the csal locus.

qPCR origin of replication.

qPCR origin of replication.

qPCR sdrD.

qPCR sdrD.

Screening for length variation in the sdrCDE locus.

Screening for length variation in the sdrCDE locus.

Screening for length variation in the SAUSA300_0293...0296 locus.

Screening for length variation in the SAUSA300_0293...0296 locus.

Construction of csal deletion cassette.

Construction of csa1 deletion cassette.

Construction of csal deletion cassette.

Construction of csa1 deletion cassette.

Screening for csa1 deletion

Screening for csal deletion

Construction of $/ p /$ deletion cassette.

Construction of $/ p /$ deletion cassette.

Construction of $/ p /$ deletion cassette.

Construction of $/ p /$ deletion cassette.

Screening for $\mid p /$ deletion

Screening for $\mid p /$ deletion

Construction of Ipp3 deletion cassette.

Construction of Ipp3 deletion cassette.

Construction of Ipp3 deletion cassette.

Construction of Ipp3 deletion cassette.

Screening for Ipp3 deletion

Screening for Ipp3 deletion

Construction of Ipp4 deletion cassette.

Construction of Ipp4 deletion cassette.

Construction of Ipp4 deletion cassette.

Construction of Ipp4 deletion cassette.

Screening for Ipp4 deletion

Screening for Ipp4 deletion

Insertion of tetK into the sdrCDE locus. Insertion of tet $K$ into the sdrCDE locus. Insertion of tetK into the sdrCDE locus. Insertion of tetK into the sdrCDE locus. Screening of tet $K$ insertion into the sdrCDE locus.

Screening of tet $K$ insertion into the sdrCDE locus.

Amplificaton of tetK from pT181.

Amplificaton of tetK from pT181. 
highly tetracycline $(\mathrm{Tc})$ resistant clones was determined by qPCR. Due to the high number of isolates to be screened, we performed the $\mathrm{qPCR}$ analysis once for each isolate. Copy numbers of strains used for further investigations were confirmed by repeating the qPCR measurement. To determine amplification frequency within a population, we interpreted a doubling in signal strength (csal/ori) as amplification of csa1. The frequency of amplifications within the culture was calculated using the formula: freq $=\frac{\text { amplifcations }\left(\frac{\mathrm{CFU}}{\mathrm{ml}}\right)}{\text { total } \mathrm{CFU}\left(\frac{\mathrm{CFU}}{\mathrm{ml}}\right)}$.

Isolation of bacterial membranes and detection of Csa1 proteins. Fractionation was carried out as described earlier ${ }^{70}$ with minor modifications. Briefly, cells were grown in TSB to stationary phase and washed once with wash buffer WB $(10 \mathrm{mM}$ Tris- $\mathrm{HCl} \mathrm{pH} \mathrm{7,10} \mathrm{mM} \mathrm{MgCl).} \mathrm{A} 1 \mathrm{ml}$ aliquot of cells adjusted to an $\mathrm{OD}_{578}=5$ was centrifuged $(18.000 \times g)$ and resuspended in $100 \mu \mathrm{l}$ digestion buffer $(10 \mathrm{mM}$ Tris$\mathrm{HCl} \mathrm{pH} \mathrm{7,10} \mathrm{mM} \mathrm{MgCl,} 500 \mathrm{mM}$ sucrose, $0.3 \mathrm{mg} / \mathrm{ml}$ lysostaphin, $250 \mathrm{U} / \mathrm{ml}$ mutanolysin, $30 \mu \mathrm{l}$ protease inhibitor cocktail (Roche -1 complete mini tablet dissolved in $200 \mu \mathrm{l} \mathrm{H}_{2} \mathrm{O}$ ), $1 \mathrm{mM}$ phenyl-methanesulfonylfluoride (PMSF). The digestion of the cell wall was carried out at $37^{\circ} \mathrm{C}$ for $1 \mathrm{~h}$ followed by centrifugation $\left(3.000 \times g\right.$ for $20 \mathrm{~min}$ at $\left.4{ }^{\circ} \mathrm{C}\right)$. The supernatant was designated "cell wall fraction". The pellet containing the protoplasts was washed with $1 \mathrm{ml} \mathrm{WB}$ (with $500 \mathrm{mM}$ sucrose) and centrifuged again as above. The protoplasts were resuspended in $200 \mu \mathrm{l}$ buffer L (100 mM Tris- $\mathrm{HCl} \mathrm{pH} 7,10 \mathrm{mM} \mathrm{MgCl}, 100 \mathrm{mM} \mathrm{NaCl}, 10 \mu \mathrm{g} / \mathrm{ml}$ DNaseI, $100 \mu \mathrm{g} / \mathrm{ml}$ RNaseA). The suspension was frozen and thawed three times to ensure complete protoplast lysis and centrifuged for $30 \mathrm{~min}\left(18.000 \times g\right.$ at $\left.4^{\circ} \mathrm{C}\right)$. The pellet (designated "membrane fraction") was washed with $1 \mathrm{ml}$ of buffer $\mathrm{L}$ and resuspended in $100 \mu \mathrm{l}$ TE buffer ( $100 \mathrm{mM}$ Tris-HCl pH 8, 1 mM EDTA). 5-15 $\mu$ l of the fractions were used for analysis by SDS-PAGE. Western immunoblotting was performed using standard procedures and mouse serum directed against Csa1C $(1: 1000)^{18}$. Secondary goat $\alpha$-mouse-DYLight 800 antibodies (LI-COR 926-32210) were used and fluorescence intensity was quantified using the "Odyssey ClX" Infrared technology from LI-COR.

Isolation of cell wall fraction and detection of SdrD proteins. The exponential cultures were inoculated from the $20 \mathrm{ml}$ overnight cultures and grown from an $\mathrm{OD}_{600}=0.1$ to 0.9 in TSB. The cell-wall fraction was isolated as described above, $10 \mu \mathrm{l}$ of sample loaded on a $7.5 \%$ gel and analyzed by Western immunoblotting using rabbit antiserum against SdrD (1:1000) (kind gift of T.J. Foster) and goat $\alpha$ rabbit-DYLight 800 secondary antibodies (LI-COR 926-32211).

Mouse bacteraemia model. Animal experiments were performed in strict accordance with the European Health Law of the Federation of Laboratory Animal Science Associations. The protocol was approved by the Regierungspräsidium Tübingen (IMIT1/17). Mice were kept in $360 \mathrm{~cm}^{2}$ (Type 2) individually ventilated cages ( 3 mice per cage) with food and water ad libidum, $12 \mathrm{~h}$ light, $22^{\circ} \mathrm{C}$ and $53 \%$ humidity. TSB medium was inoculated with $S$. aureus strains from a pre-culture and grown to mid-exponential growth phase $(2-3 \mathrm{~h})$. Bacteria were harvested, washed and diluted in sterile PBS. CFUs were determined by diluting and plating on TSA and TSA $\mathrm{TC2}_{\text {20 }}$. Six-week old female C57BL/6 wild-type mice purchased from Envigo were challenged with $1 \times 10^{7}$ live bacteria in $0.2 \mathrm{ml} \mathrm{PBS}$ injected into the tail vein. Survival and disease progression was monitored for up to $72 \mathrm{~h}$ and viable counts of bacteria in the organs were enumerated on TSA plates as well as on $\mathrm{TSA}_{\mathrm{Tc20}}$. csal copy number of up to $20 \mathrm{Tc}$ resistant colonies was determined by qPCR as described above.

Stimulation of cell lines. Stimulation of the cell lines was performed with the sterile filtered bacterial culture supernatants in TSB, diluted to a final concentration of $0.5 \%$ in a suitable medium with incubation at $37^{\circ} \mathrm{C}$ and $5 \% \mathrm{CO}_{2}$. HEK cells stably transfected with the human TLR2 gene were purchased from InvivoGen (France) and cultivated in $75 \mathrm{~cm}^{2}$ culture flasks using $20 \mathrm{ml}$ of growth medium (Dulbecco's modified eagle medium (DMEM), $10 \%$ fetal calf serum (FCS), $100 \mu \mathrm{g} \mathrm{ml}^{-1}$ normocin, and $10 \mu \mathrm{g} \mathrm{ml}^{-1}$ blasticidin). HEK cells were cultivated in DMEM, $10 \% \mathrm{FCS}$, $20 \mathrm{mM} \mathrm{L}$-glutamine, and $1000 \mathrm{u} \mathrm{ml}^{-1}$ penicillin/streptomycin. $0.3 \times 10^{6}$ cells were seeded into 24-well cell culture plates and cultivated until confluence was reached. Growth medium was then replaced by medium without FCS containing appropriately diluted stimuli and the plate was incubated for $18 \mathrm{~h}$. HL60 cells were purchased from the Leibniz Institute DSMZ-German Collection of Microorganisms and Cell Cultures (Germany) and cultivated in a $75 \mathrm{~cm}^{2}$ culture flasks using $20 \mathrm{ml}$ of growth medium (very low endotoxin-Roswell Park Memorial Institute Medium (RPMI) 1640, 10\% FCS, $2 \mathrm{mM}$ GlutaMax, $100 \mathrm{u} \mathrm{ml}^{-1}$ penicillin/streptomycin, $10 \mathrm{mM}$ 4-(2-hydroxyethyl)-1-piperazineethanesulfonic acid (HEPES)). $5 \times$ $10^{5}$ cells were seeded into U shape bottom 96-well cell culture plates and immediately stimulated with the diluted culture filtrates for $18 \mathrm{~h}$. Diluted culture filtrates exerted no toxicity towards HEK and HL60 cells as analyzed with the Cytotoxicity Detection Kit (Roche Applied Sciences). No stimulatory activity was detected in non-inoculated media at corresponding dilutions. After stimulation supernatants were collected by centrifugation for $10 \mathrm{~min}$ at $250 \times \mathrm{g}$ and stored at $-20^{\circ} \mathrm{C}$ before use. Cytokines were diluted and measured using ELISA kits (R\&D Systems) according to the manufacturer's instructions.
Stimulation of primary cells. Human PMNs were isolated from venous blood of healthy volunteers in accordance with protocols approved by the Institutional Review Board for Human Subjects at the University of Tübingen. Informed written consent was obtained from all volunteers. PMNs were isolated by standard Ficoll/Histopaque gradient centrifugation and stimulated with diluted bacterial culture filtrates (final concentration $0.25 \%$ ) in 96 -well U shaped bottom plates. $5 \times 10^{5}$ PMNs were seeded in cell culture medium (very low endotoxin RPMI 1640, $2 \mathrm{mM}$ sodium pyruvate, $2 \mathrm{mM}$ L-glutamine, $100 \mathrm{u} \mathrm{ml}^{-1}$ penicillin/ streptomycin, $10 \mathrm{mM}$ 4-(2-hydroxyethyl)-1-piperazineethanesulfonic acid (HEPES)) and incubated for $5 \mathrm{~h}$ at $37^{\circ} \mathrm{C}$ in $5 \% \mathrm{CO}_{2}$. Cytotoxicity and cytokines were measured as described above.

RecA expression. S. aureus USA300 LAC was used to inoculate $20 \mathrm{ml}$ TSB and incubated for $18 \mathrm{~h}$ at $37^{\circ} \mathrm{C} .20 \mathrm{ml}$ broth samples were inoculated to an $\mathrm{OD}_{600}=0.1$ and $0.4-2 \mu \mathrm{g} / \mathrm{ml}$ ciprofloxacin was added. The $\mathrm{OD}_{600}$ was measured each hour for $8 \mathrm{~h}$ and then after $24 \mathrm{~h}$. At $\mathrm{OD}_{600}=0.91 \mathrm{ml}$ of each culture was harvested, the cells were lysed using glass beads. Cell debris was pelleted $(17.000 \times \mathrm{g}, 10 \mathrm{~min})$ and the supernatant collected. Protein concentrations of the crude extracts were determined by Bradford and $1 \mu \mathrm{g}$ of total protein was used for SDS-PAGE and Western immunoblotting using standard procedures. Filters were incubated with rabbit $\alpha-$ RecA antibody (1:3000) (Abcam, ab63797) ${ }^{18}$ and goat $\alpha$-rabbit IgG-DYLight 800 (LI-COR 926-32211) and fluorescence intensity was quantified using the "Odyssey ClX" Infrared technology.

Statistical analysis. Statistical analysis was performed using graphpad Prism. The used tests as well as the number of replicates for each experiment are indicated in the respective figure legends.

Reporting summary. Further information on research design is available in the Nature Research Reporting Summary linked to this article.

\section{Data availability}

MinION sequencing data are deposited in the NCBI BioProject database under the accession number PRJNA601323w. The USA300 datasets analyzed here were previously deposited in the European Nucleotide archive under accession number PRJEB2870 (ref. ${ }^{15}$ ). All data obtained or analyzed in this study underlying the figures in this manuscript are available in Supplementary Data 1 or in the Source Data file. Source data are provided with this paper.

Received: 24 June 2019; Accepted: 15 June 2020;

Published online: 14 July 2020

\section{References}

1. Lindsay, J. A. Staphylococcus aureus genomics and the impact of horizontal gene transfer. Int. J. Med. Microbiol. 304, 103-109 (2014).

2. Viana, D. et al. A single natural nucleotide mutation alters bacterial pathogen host tropism. Nat. Genet. 47, 361-366 (2015).

3. Darmon, E. \& Leach, D. R. Bacterial genome instability. Microbiol. Mol. Biol. Rev. 78, 1-39 (2014).

4. Guerillot, R. et al. Unstable chromosome rearrangements in Staphylococcus aureus cause phenotype switching associated with persistent infections. Proc. Natl Acad. Sci. USA 116, 20135-20140 (2019).

5. Andersson, D. I. \& Hughes, D. Gene amplification and adaptive evolution in bacteria. Annu. Rev. Genet. 43, 167-195 (2009).

6. Sandegren, L. \& Andersson, D. I. Bacterial gene amplification: implications for the evolution of antibiotic resistance. Nat. Rev. Microbiol. 7, 578-588 (2009).

7. Elliott, K. T., Cuff, L. E. \& Neidle, E. L. Copy number change: evolving views on gene amplification. Future Microbiol. 8, 887-899 (2013).

8. Roth, A. J. Escherichia coli and Salmonella: Cellular and Molecular Biology 22 (ASM Press, 1996).

9. Anderson, P. \& Roth, J. Spontaneous tandem genetic duplications in Salmonella typhimurium arise by unequal recombination between rRNA (rrn) cistrons. Proc. Natl Acad. Sci. USA 78, 3113-3117 (1981).

10. Rocha, E. P. \& Blanchard, A. Genomic repeats, genome plasticity and the dynamics of Mycoplasma evolution. Nucleic Acids Res. 30, 2031-2042 (2002).

11. Aras, R. A., Kang, J., Tschumi, A. I., Harasaki, Y. \& Blaser, M. J. Extensive repetitive DNA facilitates prokaryotic genome plasticity. Proc. Natl Acad. Sci. USA 100, 13579-13584 (2003).

12. Laehnemann, D. et al. Genomics of rapid adaptation to antibiotics: convergent evolution and scalable sequence amplification. Genome Biol. Evol. 6, 1287-1301 (2014) 
13. Nicoloff, H., Hjort, K., Levin, B. R. \& Andersson, D. I. The high prevalence of antibiotic heteroresistance in pathogenic bacteria is mainly caused by gene amplification. Nat. Microbiol. 4, 504-514 (2019).

14. Chambers, H. F. \& Deleo, F. R. Waves of resistance: Staphylococcus aureus in the antibiotic era. Nat. Rev. Microbiol. 7, 629-641 (2009).

15. Uhlemann, A. C. et al. Molecular tracing of the emergence, diversification, and transmission of S. aureus sequence type 8 in a New York community. Proc. Natl Acad. Sci. USA 111, 6738-6743 (2014).

16. Diep, B. A. et al. Complete genome sequence of USA300, an epidemic clone of community-acquired meticillin-resistant Staphylococcus aureus. Lancet 367, 731-739 (2006).

17. Bateman, A., Holden, M. T. \& Yeats, C. The G5 domain: a potential Nacetylglucosamine recognition domain involved in biofilm formation. Bioinformatics 21, 1301-1303 (2005)

18. Schluepen, C. et al. Mining the bacterial unknown proteome: identification and characterization of a novel family of highly conserved protective antigens in Staphylococcus aureus. Biochem. J. 455, 273-284 (2013).

19. Nguyen, M. T. et al. The nuSaalpha specific lipoprotein like cluster (lpl) of S. aureus USA300 contributes to immune stimulation and invasion in human cells. PLoS Pathog. 11, el004984 (2015).

20. Shahmirzadi, S. V., Nguyen, M. T. \& Gotz, F. Evaluation of Staphylococcus aureus lipoproteins: role in nutritional acquisition and pathogenicity. Front Microbiol. 7, 1404 (2016).

21. McDevitt, D., Wann, E. R. \& Foster, T. J. Recombination at the coagulase locus in Staphylococcus aureus: plasmid integration and amplification. J. Gen. Microbiol. 139, 695-706 (1993).

22. Goerke, C., Koller, J. \& Wolz, C. Ciprofloxacin and trimethoprim cause phage induction and virulence modulation in Staphylococcus aureus. Antimicrob. Agents Chemother. 50, 171-177 (2006).

23. Stoll, H., Dengjel, J., Nerz, C. \& Gotz, F. Staphylococcus aureus deficient in lipidation of prelipoproteins is attenuated in growth and immune activation. Infect. Immun. 73, 2411-2423 (2005).

24. Nguyen, M. T. \& Gotz, F. Lipoproteins of gram-positive bacteria: key players in the immune response and virulence. Microbiol. Mol. Biol. Rev. 80, 891-903 (2016).

25. Hanzelmann, D. et al. Toll-like receptor 2 activation depends on lipopeptide shedding by bacterial surfactants. Nat. Commun. 7, 12304 (2016).

26. Cadet, J. \& Wagner, J. R. DNA base damage by reactive oxygen species, oxidizing agents, and UV radiation. Cold Spring Harb. Perspect. Biol. 5, a012559 (2013).

27. Babior, B. M. The respiratory burst of phagocytes. J. Clin. Invest. 73, 599-601 (1984).

28. Vandecraen, J., Chandler, M., Aertsen, A. \& Van Houdt, R. The impact of insertion sequences on bacterial genome plasticity and adaptability. Crit. Rev. Microbiol. 43, 709-730 (2017).

29. Heilbronner, S. et al. Genome sequence of Staphylococcus lugdunensis N920143 allows identification of putative colonization and virulence factors. FEMS Microbiol. Lett. 322, 60-67 (2011).

30. Heilbronner, S. et al. Competing for iron: duplication and amplification of the isd locus in Staphylococcus lugdunensis HKU09-01 provides a competitive advantage to overcome nutritional limitation. PLoS Genet. 12, e1006246 (2016).

31. Romero, D. \& Palacios, R. Gene amplification and genomic plasticity in prokaryotes. Annu. Rev. Genet. 31, 91-111 (1997).

32. Toussaint, J. P. et al. Gene duplication in Pseudomonas aeruginosa improves growth on adenosine. J. Bacteriol. 199, e00261-17 (2017).

33. Elde, N. C. et al. Poxviruses deploy genomic accordions to adapt rapidly against host antiviral defenses. Cell 150, 831-841 (2012).

34. Forche, A. et al. Rapid phenotypic and genotypic diversification after exposure to the oral host niche in Candida albicans. Genetics 209, 725-741 (2018)

35. Goldberg, I. \& Mekalanos, J. J. Effect of a recA mutation on cholera toxin gene amplification and deletion events. J. Bacteriol. 165, 723-731 (1986).

36. Mekalanos, J. J. Duplication and amplification of toxin genes in Vibrio cholerae. Cell 35, 253-263 (1983).

37. Cerquetti, M. et al. Presence of multiple copies of the capsulation b locus in invasive Haemophilus influenzae type b (Hib) strains isolated from children with Hib conjugate vaccine failure. J. Infect. Dis. 192, 819-823 (2005).

38. Kroll, J. S., Loynds, B. M. \& Moxon, E. R. The Haemophilus influenzae capsulation gene cluster: a compound transposon. Mol. Microbiol 5, $1549-1560$ (1991).

39. Noel, G. J., Brittingham, A., Granato, A. A. \& Mosser, D. M. Effect of amplification of the Cap b locus on complement-mediated bacteriolysis and opsonization of type b Haemophilus influenzae. Infect. Immun. 64, 4769-4775 (1996).

40. Skovgaard, O., Bak, M., Lobner-Olesen, A. \& Tommerup, N. Genome-wide detection of chromosomal rearrangements, indels, and mutations in circular chromosomes by short read sequencing. Genome Res. 21, 1388-1393 (2011).
41. Harris, S. R. et al. Genome specialization and decay of the strangles pathogen, Streptococcus equi, is driven by persistent infection. Genome Res. 25, 1360-1371 (2015)

42. Matthews, P. R. \& Stewart, P. R. Amplification of a section of chromosomal DNA in methicillin-resistant Staphylococcus aureus following growth in high concentrations of methicillin. J. Gen. Microbiol. 134, 1455-1464 (1988).

43. Gao, W. et al. Large tandem chromosome expansions facilitate niche adaptation during persistent infection with drug-resistant Staphylococcus aureus. Microb. Genomics 1, e000026 (2015).

44. Morikawa, K. et al. Expression of a cryptic secondary sigma factor gene unveils natural competence for DNA transformation in Staphylococcus aureus. PLoS Pathog. 8, e1003003 (2012).

45. Paharik, A. E. et al. The Spl serine proteases modulate Staphylococcus aureus protein production and virulence in a rabbit model of pneumonia. $m S p h e r e \mathbf{1}$, e00208-e00216 (2016).

46. Langley, R., Patel, D., Jackson, N., Clow, F. \& Fraser, J. D. Staphylococcal superantigen super-domains in immune evasion. Crit. Rev. Immunol. 30, 149-165 (2010).

47. Espinosa-Cantu, A., Ascencio, D., Barona-Gomez, F. \& DeLuna, A. Gene duplication and the evolution of moonlighting proteins. Front Genet. 6, 227 (2015).

48. Näsvall, J., Sun, L., Roth, J. R. \& Andersson, D. I. Real-time evolution of new genes by innovation, amplification, and divergence. Science 338, 384-387 (2012).

49. Bergthorsson, U., Andersson, D. I. \& Roth, J. R. Ohno's dilemma: evolution of new genes under continuous selection. Proc. Natl Acad. Sci. USA 104, 17004-17009 (2007)

50. Al-Shangiti, A. M. et al. Structural relationships and cellular tropism of staphylococcal superantigen-like proteins. Infect. Immun. 72, 4261-4270 (2004).

51. Jarraud, S. et al. egc, a highly prevalent operon of enterotoxin gene, forms a putative nursery of superantigens in Staphylococcus aureus. J. Immunol. $\mathbf{1 6 6}$ 669-677 (2001).

52. Tsuru, T. \& Kobayashi, I. Multiple genome comparison within a bacterial species reveals a unit of evolution spanning two adjacent genes in a tandem paralog cluster. Mol. Biol. Evol. 25, 2457-2473 (2008).

53. Geoghegan, J. A. et al. Role of surface protein SasG in biofilm formation by Staphylococcus aureus. J. Bacteriol. 192, 5663-5673 (2010).

54. Pollitt, E. J. G., Szkuta, P. T., Burns, N. \& Foster, S. J. Staphylococcus aureus infection dynamics. PLoS Pathog. 14, e1007112 (2018).

55. van der Veen, S. \& Tang, C. M. The BER necessities: the repair of DNA damage in human-adapted bacterial pathogens. Nat. Rev. Microbiol 13, 83-94 (2015).

56. Nguyen, M. T. et al. Lipid moieties on lipoproteins of commensal and noncommensal staphylococci induce differential immune responses. Nat. Commun. 8, 2246 (2017).

57. Nguyen, M. T. et al. Staphylococcus aureus Lpl lipoproteins delay G2/M phase transition in Hela cells. Front Cell Infect. Microbiol. 6, 201 (2016).

58. Nguyen, M. T., Peisl, L., Barletta, F., Luqman, A. \& Gotz, F. Toll-like receptor 2 and lipoprotein-like lipoproteins enhance Staphylococcus aureus invasion in epithelial cells. Infect. Immun. 86, e00343-18 (2018).

59. Shang, W. et al. beta-lactam antibiotics enhance the pathogenicity of methicillin-resistant Staphylococcus aureus via SarA-controlled lipoproteinlike cluster expression. MBio 10, e00880-19 (2019).

60. Hilmi, D. et al. Heterogeneity of host TLR2 stimulation by Staphylocoocus aureus isolates. PLoS ONE 9, e96416 (2014).

61. Barbu, E. M. et al. beta-Neurexin is a ligand for the Staphylococcus aureus MSCRAMM SdrC. PLoS Pathog. 6, e1000726 (2010).

62. Askarian, F. et al. The interaction between Staphylococcus aureus SdrD and desmoglein 1 is important for adhesion to host cells. Sci. Rep. 6, 22134 (2016)

63. Corrigan, R. M., Miajlovic, H. \& Foster, T. J. Surface proteins that promote adherence of Staphylococcus aureus to human desquamated nasal epithelial cells. BMC Microbiol. 9, 22 (2009).

64. Sharp, J. A. et al. Staphylococcus aureus surface protein SdrE binds complement regulator factor $\mathrm{H}$ as an immune evasion tactic. PLOS ONE 7, e38407 (2012).

65. Corrigan, R. M., Rigby, D., Handley, P. \& Foster, T. J. The role of Staphylococcus aureus surface protein SasG in adherence and biofilm formation. Microbiology 153, 2435-2446 (2007).

66. Viterbi, A. Error bounds for convolutional codes and an asymptotically optimum decoding algorithm. IEEE Trans. Inf. Theory 13, 260-269 (1967).

67. Kurtz, S. et al. Versatile and open software for comparing large genomes. Genome Biol. 5, R12 (2004).

68. Gruning, B. et al. Bioconda: sustainable and comprehensive software distribution for the life sciences. Nat. Methods 15, 475-476 (2018)

69. Monk, I. R., Shah, I. M., Xu, M., Tan, M. W. \& Foster, T. J. Transforming the untransformable: application of direct transformation to manipulate 
genetically Staphylococcus aureus and Staphylococcus epidermidis. MBio 3, e00277-11 (2012).

70. Heilbronner, S. et al. Competing for iron: duplication and amplification of the Isd locus in Staphylococcus lugdunensis. Plos Genet. 12, e1006246 (2016).

71. Kazakova, S. V. et al. A clone of methicillin-resistant Staphylococcus aureus among professional football players. N. Engl. J. Med. 352, 468-475 (2005).

72. Khan, S. A. \& Novick, R. P. Complete nucleotide sequence of pT181, a tetracycline-resistance plasmid from Staphylococcus aureus. Plasmid 10, 251-259 (1983).

\section{Acknowledgements}

The authors thank Timothy J. Foster for helpful discussion, editing the manuscript and for providing $\alpha \mathrm{SdrD}$ and $\alpha \mathrm{SasG}$ antiserum. We thank Libera Lo Presti for critically reading and editing this manuscript. We thank Andreas Peschel for helpful discussion. We thank GlaxoSmithKline (GSK) for providing aCsal antiserum. We acknowledge the funding support of the Research Executive Agency to $\mathrm{SH}$ (https://erc.europa.eu/). This project has received funding from the European Union's Horizon 2020 research and innovation program under the Marie Sklodowska-Curie grant agreement No "GA 655978 ". We acknowledge the support of the University of Tübingen (EKUT) as well as the Ministry for Science and Art Baden-Württemberg for support via the RiSC initiative to $\mathrm{SH}$. SH was supported by infrastructural funding from the Deutsche Forschungsgemeinschaft (DFG), Cluster of Excellence EXC 2124 Controlling Microbes to Fight Infections. MTGH was supported by Chief Scientists Office (Reference: SIRN10). None of the funding bodies was involved in the design of the study, the performance of experiments, data evaluation, writing of the manuscript or the decision about submission.

\section{Author contributions}

D.B. and A.J. performed, designed and evaluated experiments. J.P. evaluated MinION sequencing data. M.T.G.H. performed bioinformatic data curation for NGS scaffold analysis and provided assistance for data interpretation. S.H. designed the study, performed bioinformatic evaluation as well as experiments and wrote the manuscript.

\section{Competing interests}

The authors declare no competing interests.

\section{Additional information}

Supplementary information is available for this paper at https://doi.org/10.1038/s41467020-17277-3.

Correspondence and requests for materials should be addressed to S.H.

Peer review information Nature Communications thanks Minh Thu Nguyen and the other, anonymous, reviewers for their contribution to the peer review of this work. Peer reviewer reports are available.

Reprints and permission information is available at http://www.nature.com/reprints

Publisher's note Springer Nature remains neutral with regard to jurisdictional claims in published maps and institutional affiliations.

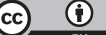

Open Access This article is licensed under a Creative Commons Attribution 4.0 International License, which permits use, sharing, adaptation, distribution and reproduction in any medium or format, as long as you give appropriate credit to the original author(s) and the source, provide a link to the Creative Commons license, and indicate if changes were made. The images or other third party material in this article are included in the article's Creative Commons license, unless indicated otherwise in a credit line to the material. If material is not included in the article's Creative Commons license and your intended use is not permitted by statutory regulation or exceeds the permitted use, you will need to obtain permission directly from the copyright holder. To view a copy of this license, visit http://creativecommons.org/ licenses/by/4.0/.

(C) The Author(s) 2020 